\title{
Las espadas en lengua de carpa - aspectos morfológicos, metalúrgicos y culturales
}

\author{
Carp's-tongue swords - morphological, metallurgical and cultural aspects
}

\author{
Dirk Brandherm $(*)$ \\ Magdalena Moskal-del Hoyo (**)
}

\section{RESUMEN}

Se presentan los resultados de un análisis de la dispersión geográfica de los distintos subgrupos morfológicos de las espadas en lengua de carpa a nivel europeo, poniéndolas en relación con una marcada variabilidad en la composición metalúrgica de estas piezas, que lleva a conclusiones inesperadas sobre la dimensión temporal y espacial de su evolución. El análisis de la variabilidad en los ritos de deposición de estos artefactos y en la composición de los conjuntos en que se encuentran, tiene importantes repercusiones sobre nuestra imagen de los contextos deposicionales de los objetos metálicos en el mundo del Bronce Final atlántico en general.

\begin{abstract}
This contribution discusses the results from a study of the spatial distribution of different morphological sub-groups of carp's-tongue swords at a European scale, relating morphological types to the metallurgial make-up of the respective pieces. From this study some surprising conclusions concerning the chronological as well as the spatial dimension of the evolution of carp's-tongue swords emerge. We also look at the degree of variability in the deposition of these artefacts and in the composition of the assemblages in question, with considerable repercussions for our understanding of the depositional contexts of metal objects in the Atlantic Bronze Age world in general.
\end{abstract}

Palabras clave: Bronce Final; Depósitos; Armas; Espadas; Metalurgia.

Key words: Final Bronze Age; Hoards; Weapons; Swords; Metallurgy.

(*) School of Geography, Archaeology and Palaeoecology. Queen's University Belfast. Belfast, BT7 1NN. Northern Ireland, UK. Correo electronico: d.brandherm@qub.ac.uk

(**) Departament de Prehistòria i Arqueologia. Universitat de València. Avgda. Blasco Ibáñez 28. 46010 València. Correo electronico: magdalena.moskal@uv.es

Recibido: 1-III-2010; aceptado: 13-IV-2010.

\section{INTRODUCCIÓN}

Las espadas en lengua de carpa constituyen uno de los principales fósiles directores del Bronce Final atlántico. Se caracterizan por una considerable variabilidad morfológica y una amplia dispersión geográfica entre Portugal y Polonia por un lado, y entre Sicilia y las Islas Británicas por el otro. En los últimos años ha quedado cada vez más claro que este grupo de armas sigue ofreciendo problemas de índole tanto cronológica como contextual que durante mucho tiempo o no se reconocieron o se daban erróneamente por resueltos. Recientemente han aparecido toda una serie de aproximaciones recientes que tenían como objetivo contribuir a su resolución. En este contexto hay que destacar particularmente los trabajos de S. Rovira $(1995,2007)$ sobre los aspectos metalúrgicos del tema, que aportaron toda una nueva dimensión a los estudios sobre las espadas en lengua de carpa y sobre la producción y circulación de objetos metálicos en el Bronce Final atlántico en general.

El objetivo principal de esta contribución es integrar mejor los datos metalúrgicos con los tradicionales enfoques tipológicos y contextuales. Al mismo tiempo queríamos aprovechar esta ocasión para presentar una primera síntesis de nuestras respectivas líneas de trabajo (Moskal 2007 (1); Brandherm 2007a; Brandherm y Burgess 2008), teniendo en cuenta también los nuevos datos aportados por la reciente obra de B. Quilliec (2007a).

(1) Este artículo está basado en la tesina de licenciatura realizada en la Universidad Jagellona de Cracovia (Uniwersytet Jagielloński) bajo la dirección de Prof. Dr. M. Gedl: M. Moskal, Schytkowobrazowe miecze z językiem karpia w Europie, 2003. 
Sin querer aparecer demasiado empiricistas, consideramos evidente que un detallado análisis morfológico y una pormenorizada caracterización metalúrgica de los ítems en cuestión son condiciones necesarias para efectuar estudios con valor informativo pertinente sobre las cadenas operativas que intervienen en la fabricación, el empleo y la deposición de las espadas del Bronce Final (Quilliec 2007b). Al mismo tiempo, un análisis del contexto arqueológico de estas armas y de la composición de los depósitos del llamado "complejo de lengua de carpa", a nuestro entender, resulta imprescindible para cualquier interpretación de su función social y posible simbolismo. Por ello en esta contribución desarrollaremos un poco más lo que ya habíamos dicho al respecto. La falta de espacio aquí nos obligada a dejar fuera otra serie de aspectos importantes sobre las espadas y los depósitos del Bronce Final.

\section{TIPOLOGÍA}

Hace más de medio siglo H.N. Savory (1949: 138) recalcó la falta de uniformidad morfológica en las espadas en lengua de carpa. También J. Cowen (1971: 154), en su ya clásico artículo sobre las espadas del tipo Monte Sa Idda, advirtió que "la gran familia de las espadas en lengua de carpa" incluía claramente toda una serie de tipos y variantes distintos. Unos años después, A. Jockenhövel (1980: 120) señaló la variabilidad morfológica de este grupo de armas y resaltó la necesidad de someterlas a una clasificación tipológica más rigurosa. Desde entonces, varios autores han propuesto criterios para tal clasificación, muchas veces centrándose en la ausencia o presencia y en la forma de los recazos, así como en la morfología de la guarda (p. e. Meijide 1988: 33, 39; Giardino 1995: 194). Nosotros también hemos experimentado con los distintos criterios, y los resultados de una clasificación según la forma de los recazos han sido bastante similares, pero no idénticos, a los de una clasificación según la forma del enmangue. Esta última produce grupos algo más homogéneos en cuanto a su distribución geográfica y a sus aspectos cronológicos (compárense Brandherm 2007a: Láms. 47, 48; Brandherm y Burgess 2008: Figs. 3, 5, 9; Moskal 2007: Fig. 9).

En el presente trabajo, por lo tanto, como criterio principal para nuestra clasificación tipológica emplearemos la forma del enmangue, siguiendo un esquema ya introducido en una ocasión anterior (Brandherm y Burgess 2008: 136-151). Según la sistemática de este esquema se distinguen tres tipos principales de espadas en lengua de carpa: Huelva, Nantes y Monte Sa Idda (Fig. 1: a, c, d). El nombre del primer tipo deriva de las conocidas armas del conjunto dragado de
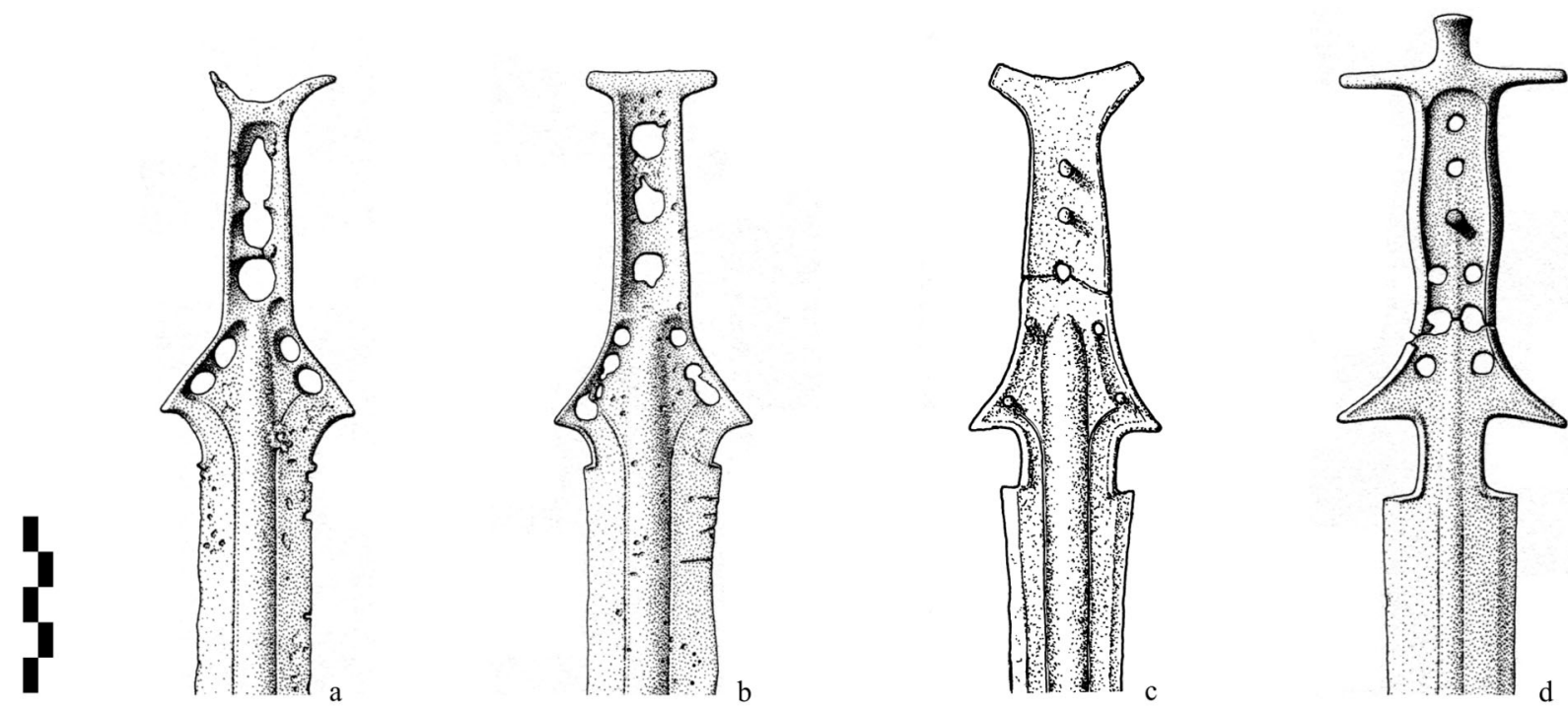

Fig. 1. a tipo Huelva; b grupo transicional; c tipo Nantes; d tipo Monte Sa Idda - (a Ría de Huelva, según Brandherm 2007a: Lám. 9, 45; b Ría de Huelva, según Brandherm 2007a: Lám. 9, 50; c Saint-Yrieux "Vénat”, según Coffyn et al. 1981: Lám. 4, 19; d Villaverde del Río, según Brandherm 2007a: Lám. 28, 172). 
la Ría de Huelva (Ruiz-Gálvez 1995), mientras que el del tipo Nantes procede de dos depósitos importantes de dicha localidad francesa: la Prairie de Mauves (Briard 1966) y el Jardín de Plantes (Briard 1971). Finalmente, el tipo Monte Sa Idda, ya definido como tipo sui generis por Cowen (1971: 155), se corresponde con el epónimo depósito sardo (Taramelli 1921).

Esta terminología tiene la ventaja que los respectivos conjuntos epónimos contienen casi la totalidad de las variantes que luego se pueden distinguir en cada uno de los tres tipos. Otros tipos de espadas en lengua de carpa, como p. e. Vénat o Safara, definidos anteriormente (Cowen 1971: 154-155; Meijide 1988: 39, 54; Brandherm 2007a: 88-90), aquí se incluirán como subgrupos dentro del tipo Nantes, ya que tan sólo se diferencian de las demás piezas de este tipo por algún detalle muy concreto de la empuñadura (Fig. 4: c, d).

La transición entre el huso y la guarda distingue el tipo Huelva del tipo Nantes. En el tipo Huelva resulta más bien angular, mientras que en el tipo Nantes forma una curvatura bastante suave (Fig. 1: a, c). Existe un pequeño grupo de piezas intermedio, donde esa transición ya no es muy angular sino más bien curvada, pero que no exhiben la curvatura suave que caracteriza el tipo Nantes (Fig. 1: b). Por otro lado, el tipo Monte Sa Idda en general también luce una transición curvada entre el huso y la guarda, muchas veces destacada por un ligero estrangulamiento en la parte inferior del huso (Fig. 1: d).

En cada uno de estos tipos se puede individualizar una serie de variantes, que caracterizaremos brevemente, ya que con la ampliación del catálogo se han producido modificaciones importantes desde que se presentó la primera versión de este esquema (Brandherm y Burgess 2008: 137-147).

Tipo Huelva: teniendo en cuenta la totalidad de las piezas respectivas a nivel europeo, dentro del tipo Huelva se pueden diferenciar los siguientes subgrupos:

Variante Cambes: se caracteriza por calados tanto en el huso como en la guarda. Los dos lados del huso son ligeramente convexos, resultando en un contorno algo hinchado, que termina en cola de pez. Algunos ejemplares disponen de recazos que no suelen ser muy pronunciados (Fig. 2: a). Como ya se expuso anteriormente (Brandherm y Burgess 2008: 138 nota 41), estas piezas están estrechamente relacionadas con las espadas del tipo

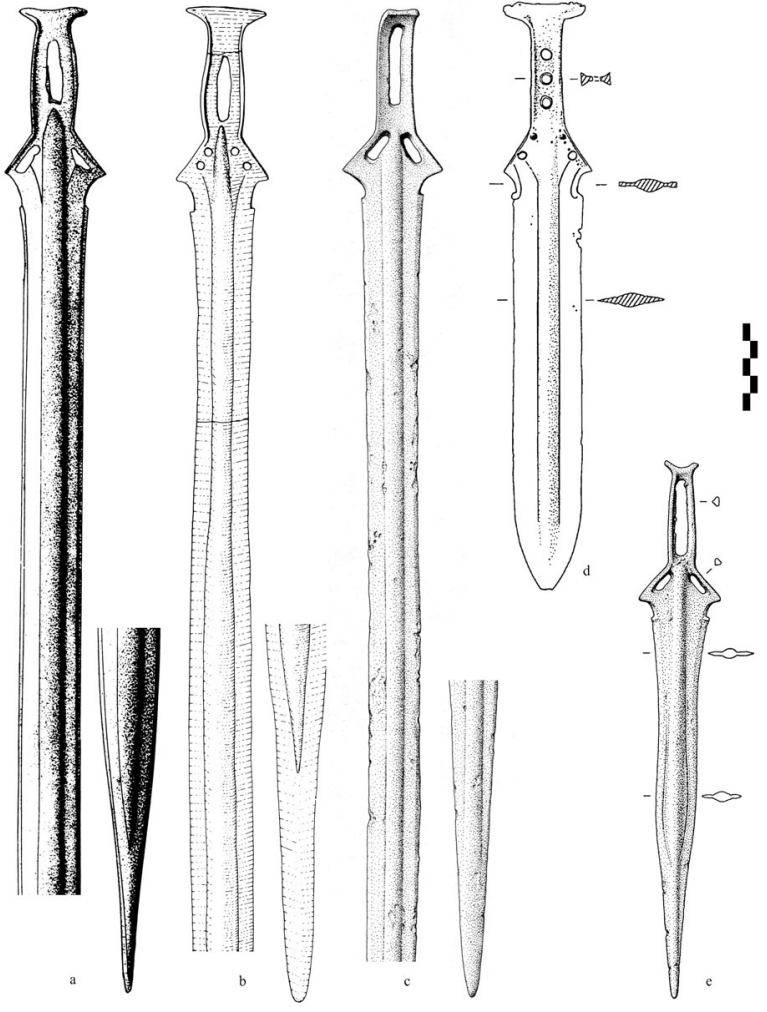

Fig. 2. Variantes del tipo Huelva según las piezas epónimas: a Cambes; b Mouy; c Marmolejo; d Oissel; e Puertollano - (a según Coffyn 1985: Fig. 48, 1; b según Blanchet 1984: Fig. 170, 3; c según Brandherm 2007a: Lám. 16, 86; d según Billard et al. 1993, Fig. 14, 5; e según Brandherm 2007a: Lám. 24, 149).

Cordeiro del ámbito ibérico y del suroeste de Francia. A excepción de dos piezas halladas en el alto Rhin y en Cerdeña, su dispersión queda restringida a la Península Ibérica y a Francia al sur del río Loira (Fig. 3).

Variante Mouy: este grupo ha sido descrito previamente como "otras espadas del tipo Huelva con el huso hinchado" (Brandherm y Burgess 2008: 138). Están estrechamente relacionadas con la variante Cambes, con la que comparten esa forma del huso que en las piezas de la presente variante suele terminar en cola de pez o en abanico. Se diferencian de la variante Cambes en que las piezas tienen orificios de remaches en el huso y/o en la guarda, lo que resulta en un carácter algo más heterogéneo del grupo, y en que los hombros de la guarda, en general, son más inclinados, mientras que los recazos son un poco más pronunciados (Fig. 2: b). La dispersión geográfica de la variante Mouy resulta totalmente distinta 
a la variante Cambes. Queda limitada al norte de Francia, con tan sólo una pieza hallada más al sur, en las orillas del lago de Ginebra (Fig. 3).

Variante Marmolejo: las piezas de este subgrupo se han designado con anterioridad como "otras espadas del tipo Huelva con el huso con lados paralelos" (Brandherm y Burgess 2008: 138). Tanto en la guarda como en el huso tienen calados. El huso suele terminar en cola de pez o, en algún caso, en forma de T (Fig. 2: c). A veces el calado del huso está dividido en dos. En este caso no siempre es fácil distinguirlo de un huso con tres perforaciones grandes y rectangulares -rasgo típico de la variante Oissel- que ha sufrido algún defecto de fundición o algún daño posterior. Menos un hallazgo suizo, todas las espadas de este grupo proceden del ámbito peninsular (Fig. 3).

Variante Oissel: las respectivas piezas se clasificaron anteriormente como "espadas del tipo Huelva con huso en barra" (Brandherm y Burgess 2008: 138), estrecho, con lados paralelos y con tres orificios individuales para remaches. En la guarda aparecen los orificios individuales para remaches y los calados. Los recazos también son bastante variables. Por lo general, esta variante tiene el pomo en forma de $\mathrm{T}$ y los orificios para remaches suelen ser mayores que en la variante Mouy. En algunos casos dos de las perforaciones del huso quedan unidas por un defecto de fundición o alguna modificación secundaria, dejando y no siempre se diferencian fácilmente de los ejemplares menos típicos de la variante Marmolejo. La morfología de las espadas de la variante Oissel procedentes de la Ría de Huelva varía más que en el resto del subgrupo: los husos pueden tener un ligerísimo estrechamiento o hinchamiento y terminan con mayor frecuencia en cola de pez (Fig. 2: d). La dispersión de esta variante se limita a Andalucía occidental y al norte de Francia, salvo un hallazgo procedente del centro de Alemania (Fig. 3).

Variante Puertollano: se trata de espadas cortas que lucen todos los rasgos característicos del tipo Huelva, salvo el tamaño reducido, a la mitad de la longitud normal de otras espadas en lengua de carpa. La empuñadura de los ejemplares conocidos lleva un calado en el huso y dos en la guarda, como las piezas de la variante Cambes (Fig. 2: e). En la variante Puertollano no se incluyen las piezas cuyo tamaño final con seguridad se debe a la modificación secundaria de una espada con dimensiones normales, caso de la pieza epó- nima de la variante Oissel (Fig. 2: d). La dispersión de la variante Puertollano queda limitada a la Península Ibérica, a excepción de una pieza hallada en el suroeste de Francia (Fig. 3).

En primer lugar resaltamos que, en la presente clasificación de todo el material a escala europea, se está empleando un enfoque monotético en vez del enfoque politético utilizado previamente en la clasificación de las espadas peninsulares del tipo Huelva y que permitió individualizar hasta tres series distintas dentro de este tipo (Brandherm 2007a: 57-59). No se trata de una modificación de ideas por nuestra parte, como insinuó A. Mederos (2008: 44), sino más bien de un ajuste a las distintas trayectorias evolutivas del tipo Huelva en diferentes áreas geográficas. De hecho, ya desde el inicio se advirtió que el enfoque politético aplicado al ámbito peninsular no se debería ampliar a las espadas extrapeninsulares (Brandherm 2007a: 87). No obstante, eso de ninguna manera implica que los resultados de nuestra primera aproximación estuviesen superados ya con respecto a su propio marco de referencia.

Al contemplar la dispersión de los distintos subgrupos del tipo Huelva, llama la atención que las variantes Cambes, Marmolejo y Puertollano, todas con empuñadura con tres calados, faltan al norte de Francia más allá del río Loira. La gran mayoría efectivamente procede del ámbito peninsular. Por el contrario, las variantes Mouy y Oissel, que lucen orificios individuales para remaches o una combinación de orificios y calados, tienen su mayor dispersión al norte de los Pirineos. Consecuentemente, las diferencias observadas en el predominio de una u otra solución técnica del montaje de la empuñadura (orificios versus calados) parecen indicar unas claras preferencias geográficas. Sin embargo, resulta muy difícil averiguar si detrás de estas discrepancias existe también una evolución cronológica, como recientemente ha propuesto Mederos (2008: 58).

En un trabajo anterior (Brandherm 2007a: 57-58) las espadas peninsulares del tipo Huelva se han clasificado utilizando los mismos elementos de la empuñadura junto con el tipo de recazos como posibles indicadores de unos rasgos arcaicos, intermedios o evolucionados. El carácter arcaico o evolucionado de algunos de esos rasgos ha generado ciertas dudas (Mederos 2008: 44- 58). Tales problemas y nuestras propias reservas ya se expusieron con anterioridad (Brandherm 2007a: 57-58). De cualquier forma, insistimos en que, en su momento, 


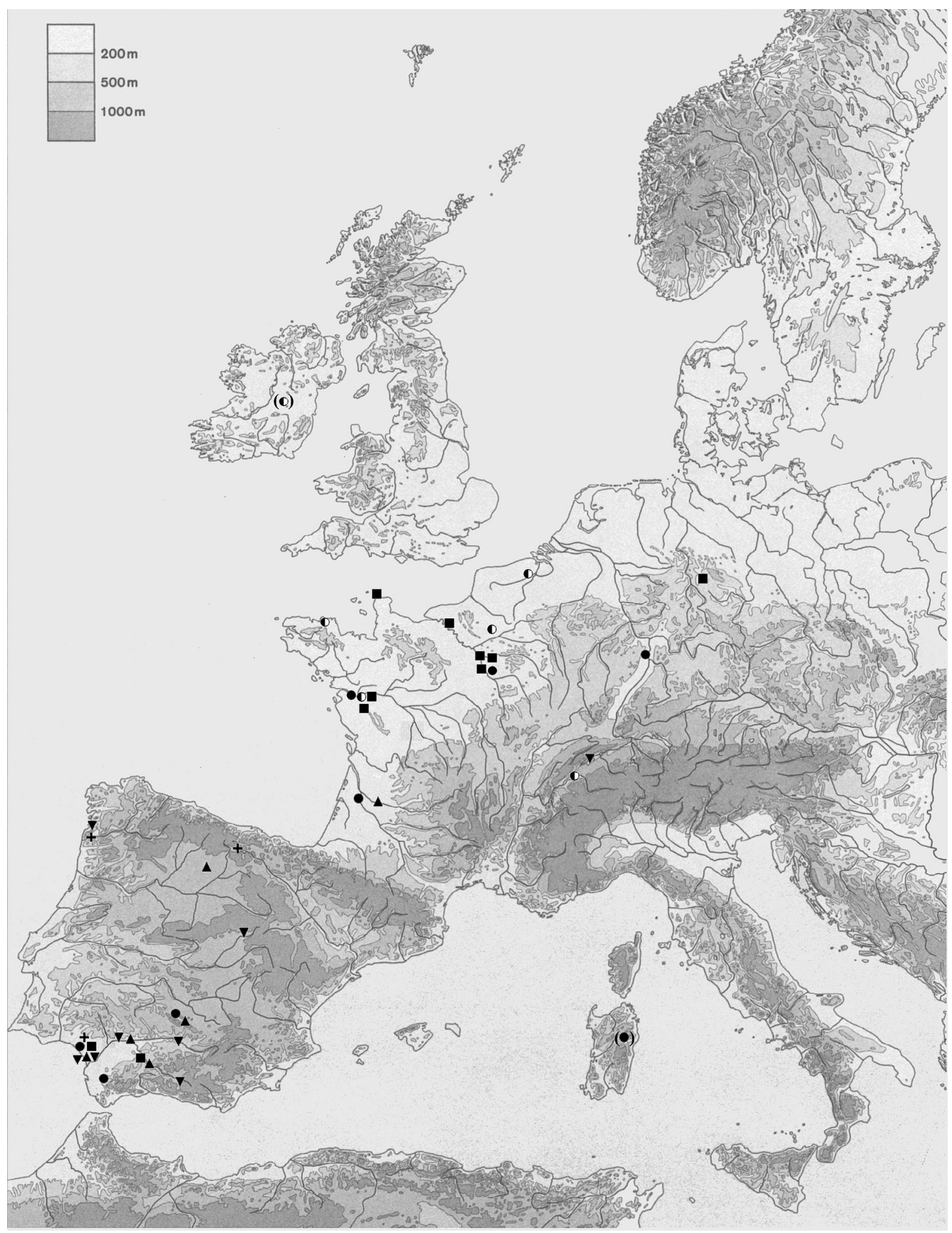

Fig. 3. Dispersión geográfica del tipo Huelva: variante Cambes $=\bullet$; variante Mouy $=\mathbf{O}$; variante Marmolejo $=\boldsymbol{\nabla}$; variante Oissel $=\mathbf{\square}$; variante Puertollano $=\mathbf{\Delta}$; variante sin determinar $=\boldsymbol{+}-$ (según Brandherm y Burgess 2008: Fig. 3 [actualizado]). 
no se emplearon los términos "arcaico" y "evolucionado" como simples sinónimos de "antiguo" y "reciente". Tampoco las distintas series de piezas que, entonces, se distinguieron dentro del tipo Huelva se pueden traducir sin más en "fases" cronológicas (contra Mederos 2008: Tab. 1).

La casi total ausencia de conjuntos cerrados con espadas del tipo Huelva no facilita seriación cronológica. El depósito de Hío, que incorpora una espada de la variante Marmolejo, claramente se debe sincronizar con un momento tardío de la fase británica Wilburton, mientras que la mayoría de los materiales de la Ría de Huelva parece corresponder ya a la fase Blackmoor (Brandherm 2007a: 83-84; Burgess y O'Connor 2008: 48-49; Gerloff 2010: 203-204).

Finalmente, en la Ría de Huelva aparecen todas las variantes del tipo Huelva, menos la variante Mouy. En este caso, resulta tentador interpretar el número de espadas de cada variante como evidencia de una sucesión (Cambes: 3 piezas; Marmolejo: 16 piezas; Oissel: 22 piezas). De este modo, las variantes más antiguas podrían ser menos corrientes en el momento de deposición de los objetos. De todas formas, nunca se debe olvidar que el lote de piezas dragadas en la Ría de Huelva no constituye un conjunto cerrado, lo que limita bastante el valor informativo de esa variación cuantitativa que siempre puede deberse a factores ajenos a la cronología (Brandherm 2007b: 295). En fin, aunque es difícil establecer una secuencia cronológica de las distintas variantes del tipo Huelva, queda muy claro que dicho tipo fue utilizado durante bastante tiempo.

Lo que resulta indudable es la mayor antigüedad del tipo Huelva en comparación con las espadas en lengua de carpa "clásicas" del tipo Nantes y con los depósitos del llamado "complejo de lengua de carpa" del norte de Francia y sudeste de Inglaterra (Brandherm y Burgess 2008: 142). Hacemos hincapié otra vez en que, en la actualidad, no se conoce ninguna espada tipo Huelva procedente de un depósito de dicho grupo, mientras que las espadas del tipo Nantes constituyen uno de sus elementos más característicos. No disponemos de dataciones radiométricas procedentes directamente de conjuntos cerrados con espadas en lengua de carpa, pero las dataciones por radiocarbono de unos restos de astiles de lanza dragados en la Ría de Huelva por un lado, y las dataciones radiométricas de elementos atribuibles a la fase británica Ewart Park por el otro, resultan consistentes con la idea de una sucesión cronológica entre el tipo Huelva y el tipo Nantes. Esta podría situarse en la segunda mitad del siglo X o en los inicios del siglo IX cal a.C. (Needham et al. 1997: 76-80; Brandherm 2007a: 176-177).

Más arriba ya advertimos que existe un grupo relativamente pequeño de espadas cuyas empuñaduras tienen características intermedias (Brandherm y Burgess 2008: 148 lista 16). Desconocemos si, realmente, corresponden a un horizonte cronológico de transición o si nos enfrentamos con un simple fenómeno de variabilidad morfológica sincrónica. Habla en favor de la primera posibilidad que todas estas piezas tengan huso en barra, asemejándose a la variante Oissel, de la cual no siempre son fácilmente distinguibles. Por otro lado, una curvatura liviana entre el huso y la guarda se produce con mucha más facilidad en un huso de lados paralelos o subparalelos que en una variante con huso hinchado. En todo caso no deja de ser llamativo que tres de las piezas en cuestión se hallaran en depósitos del "complejo de lengua de carpa" (Gouesnach "Menez Tosta", La Grimaudiere "Notre-Dame-d'Or", Saint-Yrieix-surCharente "Vénat"). Además, frente a las espadas del tipo Huelva claramente diferenciadas, algunos de los ejemplares de este grupo proceden de localidades británicas (Fig. 8). Por estas razones nos inclinamos a interpretar la mayoría como indicadores de la transición al tipo Nantes también en un sentido cronológico.

Igualmente, cualquier tentativa de determinar el área de formación del tipo Huelva tropieza con serios problemas. Aunque mucho habla en favor del sur de la Península Ibérica, tampoco se puede excluir un posible origen norpirenaico. Actualmente resulta imposible determinar si la elevada variabilidad morfológica que se observa en la variante Mouy se debe a la presencia de otros tipos de espadas en el norte de Francia en el momento de introducirse el tipo Huelva o si indica un estadio formativo del tipo Huelva en su área de origen (2). Tampoco podemos descartar por completo la posibilidad de un origen en Cerdeña. Allí claramente antes del nacimiento del tipo Huelva, existen hojas de espadas con filos rectos y el característico nervio central, que cuentan entre los

(2) Para indicar el posible origen francés de estas espadas también se ha propuesto la designación tipo Saint-Philbert (Burgess y O'Connor 2008: 52). Para evitar posibles confusiones aquí vamos a mantener la denominación convencional como tipo Huelva, sin presuponer de su origen efectivo. 
prototipos más convincentes de las subsiguientes hojas de las espadas en lengua de carpa (véase p. e. Begemann et al. 2001: Fig. 2).

Tipo Nantes: su área de origen con casi toda seguridad se debe buscar en el norte de Francia o sudeste de Inglaterra, el área de dispersión de las piezas con empuñadura "transicional" y el núcleo del "complejo en lengua de carpa". Dentro de este tipo diferenciamos cuatro variantes principales:

Variante Amboise: comprende espadas con el huso hinchado, de lados algo convexos. Comparten este rasgo con la gran mayoría de las espadas atlánticas del Bronce Final y, por tanto, no nos atrevemos a defender que este subgrupo descienda directamente de una de las variantes con huso hinchado del tipo Huelva en concreto, máxime cuando todas las piezas del grupo "transicional" lucen huso de barra. Un cierto número de piezas de la variante lleva calados en el huso, pero raramente en la guarda. En general, los orificios individuales en este subgrupo y los recazos pronunciados aparecen con mayor frecuencia (Fig. 4: a). $\mathrm{Su}$ dispersión geográfica resulta bastante amplia, desde Inglaterra hasta el sur de Francia y desde la Bretaña francesa hasta Polonia, con una clara concentración de la mayoría de las piezas en el norte y el oeste de Francia (Fig. 5).

Variante Challans: su rasgo diagnóstico es el huso de lados paralelos o ligeramente convergentes hacia el pomo (Fig. 4: b). Varía considerablemente la anchura del huso y las piezas con el huso más estrecho -en barra- se asemejan mucho a las empuñaduras de nuestro grupo "transicional". Como ya se resaltó (Brandherm y Burgess 2008: 143), un porcentaje significativo de ejemplares de la variante Challans con esta forma particular de huso aparecieron como hallazgos aislados. Con todas las debidas reservas este hecho quizás pudiera ser interpretado como indicativo de una cronología relativamente temprana de las respectivas piezas dentro de la trayectoria evolutiva de la presente variante. Como hallazgos aislados de este subgrupo faltan por completo en Inglaterra, donde también aparecen ejemplares con huso muy estrecho, no nos parece oportuno poner demasiado peso en la relativa anchura del huso como indicador cronológico. Llama la atención la dispersión más restringida de la presente variante frente a la variante Amboise, fenómeno que ya hemos observado en las respectivas variantes del tipo Huelva con huso de lados paralelos por un lado y huso hinchado por el otro. En

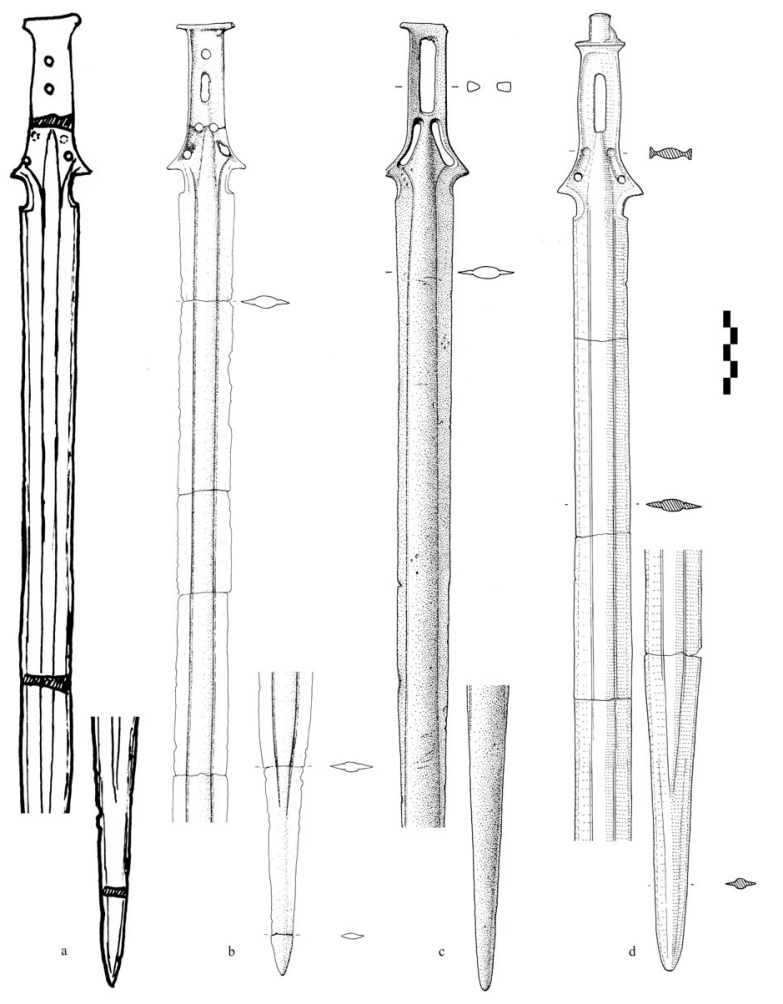

Fig. 4. Variantes del tipo Nantes según las piezas epónimas: a Amboise; b Challans; c Safara; d Saint-Yrieix "Vénat" - (a según Bastien 1966: Fig. 3; b según Verney 1990: Fig. 1, 4; c según Brandherm 2007a: Lám. 26, 164; d según Coffyn et al. 1981: Lám. 4, 1).

ambos tipos la dispersión de espadas con huso de lados rectos resulta mucho menos amplia que la de piezas con huso hinchado, ya que se centra en el norte y oeste de Francia, y en el caso del tipo Nantes también en el sur de Inglaterra (Fig. 5).

Variante Safara: individualizado inicialmente por Ruiz-Gálvez (1984: 271), y definido en ocasiones posteriores como tipo sui generis (Meijide Cameselle 1988: 39; Brandherm 2007: 88), en la presente clasificación tipológica este subgrupo se redefine como variante del tipo Nantes. Consta de un limitado número de piezas características por una empuñadura con tres calados. Los dos ubicados en la guarda están desplazados hacia el tercio distal del huso. El pomo tiene forma de $\mathrm{T}$, con apéndices laterales que pueden resultar algo atrofiados. Los recazos suelen ser bastante pronunciados (Fig. 4: c). Alguna pieza de esta variante luce un nervio central muy estrecho, rasgo que la relaciona con el tipo Monte Sa Idda (p. e. Brandherm 2007: 89 no. 166). Los ejemplares de la variante Safara conocidos en la actualidad se halla- 


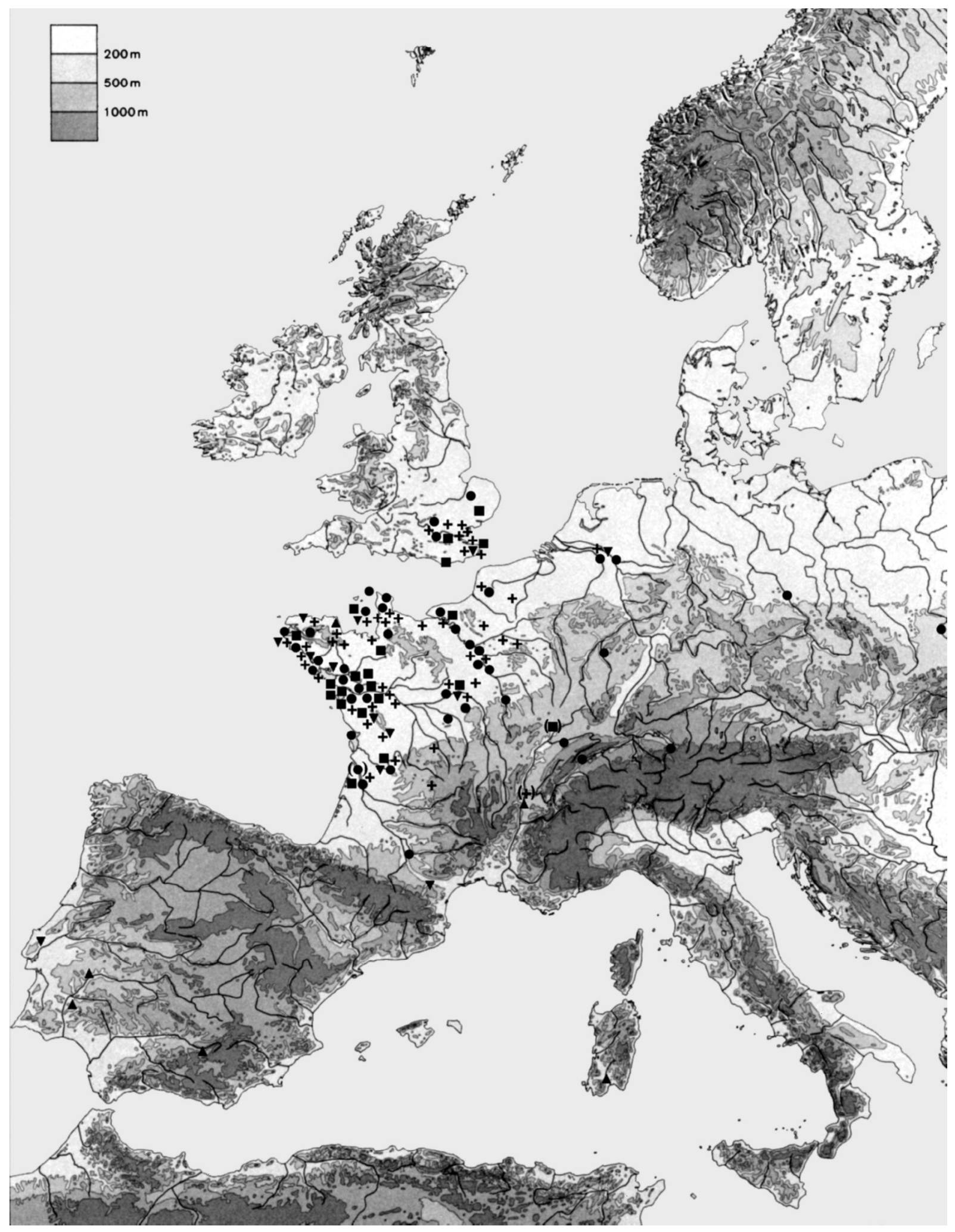

Fig. 5. Dispersión geográfica del tipo Nantes: variante Amboise $=\boldsymbol{\bullet}$; variante Challans $=\mathbf{\square}$; variante Safara $=\mathbf{\Lambda}$; variante Vénat $=\boldsymbol{\nabla}$; variante sin determinar $=\boldsymbol{+}-($ según Brandherm y Burgess 2008: Fig. 5 [actualizado] $)$.

T. P., 67, N. ${ }^{\circ} 2$, julio-diciembre 2010, pp. 431-456, ISSN: 0082-5638 doi: $10.3989 /$ tp.2010.10049 
ron en el sur de la Península Ibérica, salvo dos procedentes de la Bretaña francesa y de Cerdeña. Por lo tanto, su dispersión geográfica encaja muy bien con la imagen general de una concentración de las espadas en lengua de carpa con empuñadura de tres calados principalmente en el ámbito ibérico peninsular y en el sur de Francia (Fig. 5).

Variante Vénat: individualizado ya como un tipo propio (Cowen 1971: 154; Meijide 1988: 54; Brandherm 2007: 90), en nuestra clasificación es considerado una variante del tipo Nantes. Sólo la forma peculiar del pomo permite distinguirlo de las demás variantes de este tipo (Brandherm y Burgess 2008: 146). A diferencia de otras espadas del tipo Nantes, el pomo es macizo, cilíndrico o ligeramente ovalado, rematado en botón. El origen de esta forma del pomo claramente se encuentra en las espadas de empuñadura maciza de los Campos de Urnas tardías de Europa Central (Brandherm y Sicherl 2001: 231-236). Por eso no resulta nada sorprendente que entre las espadas con hoja en lengua de carpa y empuñadura maciza o semimaciza haya varios ejemplares estrechamente relacionados con la variante Vénat. Algunas de las respectivas piezas se habían incluido previamente también en el "tipo" Vénat según la definición sensu lato (Brandherm 2007: 91 nos. 168-170), pero teniendo en cuenta la totalidad del material francés, parece mucho más oportuno restringir la definición a este grupo. Igual que la mayoría de las demás espadas del tipo Nantes, las de la variante Vénat suelen presentar recazos muy marcados (Fig. 4: d). El área de dispersión coincide, en general, con la mayor concentración del tipo Nantes. Tan sólo una pieza se halló en el ámbito peninsular (Fig. 5).

La gran mayoría de las espadas del tipo Nantes proceden de los depósitos del "complejo de lengua de carpa". Debido a la fragmentación de los materiales en los mismos -muy superior, p. e., a la de los materiales dragados de la Ría de Huelva- en un buen número de piezas resulta imposible determinar la variante a que pertenecen. Por ello es difícil cuantificar las distintas variantes en determinados conjuntos. Por otra parte, basándose en la frecuente asociación de las variantes Amboise, Challans y Vénat, se puede afirmar su contemporaneidad general. La variante Safara por el momento falta en los depósitos de este grupo, debido con toda probabilidad no tanto a factores cronológicos cuanto a su casi total ausencia en el área de dispersión del "complejo de lengua de carpa". No obstante, el ejemplar del depósito sardo de Decimoputzu "Monte Sa Idda" indica su contemporaneidad con las demás variantes del tipo Nantes. También en este sentido es muy importante la pieza con características híbridas de la variante Safara y del tipo Monte Sa Idda del depósito portugués de Carvalhal "Fiéis de Deus" que, por el sobremoldeo de un pomo macizo en un momento posterior, se convirtió en un ejemplar de la variante Vénat (Fig. 6: d).

Tipo Monte Sa Idda: fue individualizado por Cowen (1971: 155-156) por sus recazos extremamente pronunciados y el pomo con aletas laterales atrofiadas, rematado en botón. Luego se amplió la lista de criterios característicos de este tipo (Brandherm 2007: 93-94), diferenciándose las siguientes variantes:

Variante Villaverde del Río: se distingue por la forma subrectangular de los recazos (Fig. 6: a).

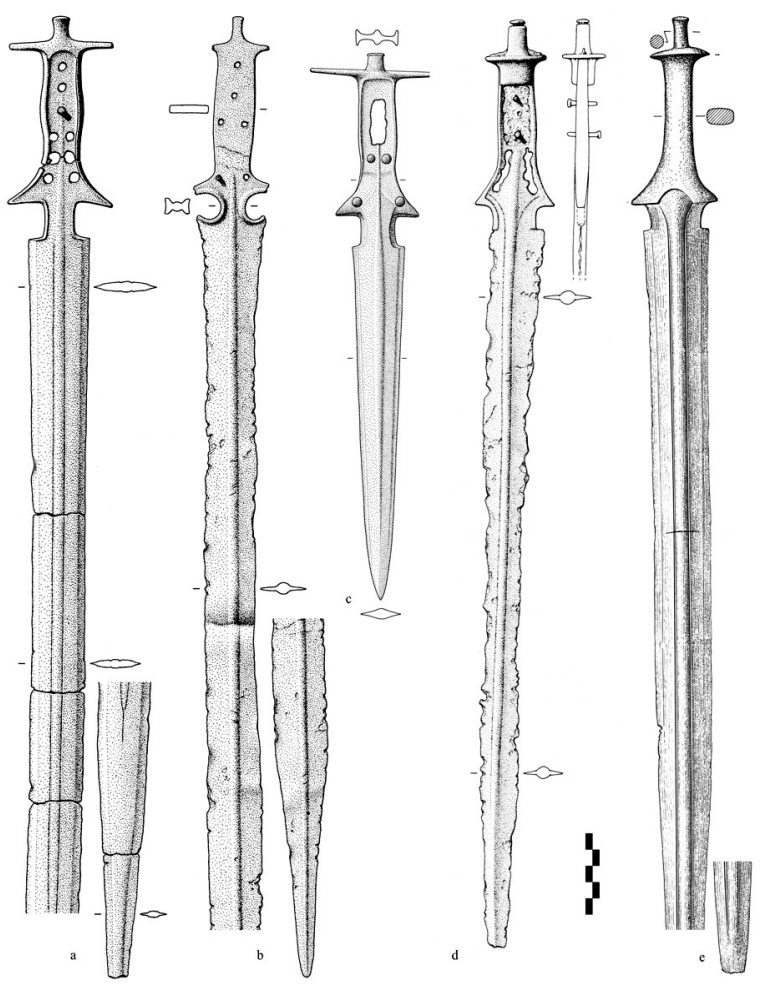

Fig. 6. Variantes del tipo Monte Sa Idda (a-c) y espadas en lengua de carpa imitando modelos centroeuropeos con empuñadura semimaciza (d) y maciza (e): a Villaverde de Río; b Alcalá del Río; c Gironde (variante Boom); d Fiéis de Deus; e alrededores de Saumur - (a. b según Brandherm 2007a: Lám. 28, 172. 173; c según Gallay 1988: Lám. 47, 1435; d según Brandherm 2007a: Lám. 27, 167; e según Cordier y Gruet 1975: Fig. 46, 3). 
Incluye un número muy limitado de piezas, en su mayoría sardas, aunque también del sur de la Península Ibérica e Italia (Fig. 7).

Variante Alcalá del Río: se definen por la forma de los recazos desmesurados que tienden a cerrarse en semicírculo y, a veces, casi en círculo (Fig. 6: b). Los ejemplares de la variante Alcalá son más numerosos que los de la variante Villaverde. En su mayoría proceden del sur peninsular, aunque en Cerdeña también las hay (Fig. 7). Además, en Ronda (Málaga) se ha documentado un molde que claramente demuestra su producción en la Península Ibérica (Brandherm 2007: 96 no. 178).

Variante Boom: comprende principalmente espadas cortas o incluso puñales. Además de las dimensiones muy reducidas de la hoja destacan las aletas en el pomo, que son más macizas que en otras variantes de este tipo, y la guarda menos ancha (Fig. 6: c). Es un grupo poco numeroso, con un posible hallazgo en Bélgica y dos piezas de procedencia poco segura del sur y del oeste de Francia (Fig. 7). Ninguna de estas piezas procede de un contexto bien definido, por lo que Cowen (1971: 157) puso en duda su autenticidad como armas del Bronce Final. Por el momento, lo más prudente es seguir considerándolas piezas sospechosas sin utilizarlas para sacar conclusiones importantes.

No resulta fácil identificar el origen del tipo Monte Sa Idda y por ahora parece imposible determinar si piezas como la de Almargen "Casablanca" (Brandherm 2007: 59 no. 44) con empuñadura "transicional" y recazos muy anchos forman una de sus raíces. Tanto Cerdeña como el sur de la Península Ibérica apuntan como posibles cunas de este grupo morfológico. En cambio, la cronología del tipo parece bien establecida, gracias a toda una serie de conjuntos cerrados que lo incluyen.

El depósito de Populonia "Falda della Guardiola", con un fragmento de una pieza de la variante Villaverde del Río, debe fecharse en el Primo Ferro $2 B$ (Giardino 1995: 54 nota 148). Una cronología idéntica se puede establecer para el conjunto epónimo de Decimoputzu "Monte Sa Idda", que contiene fragmentos tanto de la variante Villaverde como Alcalá del Río, junto a un ejemplar de la variante Safara del tipo Nantes. Entonces habrá que considerar dichos depósitos coetáneos con conjuntos tardíos del "complejo de lengua de carpa", como Saint-Yrieix-sur-Charente "Vénat", y también con la fase Ha Cla de Centroeuropa (Ruiz-Gálvez 1986: 14). Los dos depó- sito sardos de Nuragus "Forraxi Nioi" y Teti "Abini", ambos con fragmentos de ejemplares de la variante Villaverde del Río, difícilmente pueden ser mucho más antiguos (Giardino 1995: 49).

En el ámbito peninsular dos ejemplares de la variante Alcalá del Río se conocen en contextos funerarios, algo por sí mismo muy indicativo de una cronología bastante tardía. La tumba de Villaverde del Río, lamentablemente, está muy mal documentada (Ruiz-Gálvez 1984: 133-134), pero como ritos funerarios detectables en el registro arqueológico en la zona sólo reaparecen con la influencia fenicia, incluso con una cronología "alta" para el inicio de la presencia fenicia en Occidente (Brandherm 2008: 115-126) una fecha anterior a finales del siglo IX a.C. resulta muy poco probable. La pieza procedente de la tumba orientalizante de Cástulo -significativamente el único ejemplar de hierro del tipo Monte Sa Iddano se puede fechar mucho antes de mediados del siglo VII a.C., igualmente aceptando una cronología "alta" para el fenómeno orientalizante. Partiendo de una cronología algo más "baja", también se ha defendido su datación en el último cuarto del siglo VII a.C. (Jiménez Ávila 2002: 242).

En resumen, la cronología del tipo Monte Sa Idda parece cubrir como mínimo el intervalo cronológico entre mediados/finales del siglo IX y mediados del siglo VII a.C. En el mismo intervalo, con toda probabilidad, habría que colocar la espada corta con hoja en lengua de carpa y empuñadura atípica del depósito de Ocenilla, asociada a una punta de lanza de bronce tipo Llyn Fawr (Almagro Basch 1940: Fig. 21), así como una espada corta muy similar de hierro, procedente de una tumba hallada en El Atance "Las Horazas" (Schüle 1969: Lám. 12, 7).

Finalmente, tanto en la Península Ibérica como en la Itálica existen armas que lucen hojas con punta "en lengua de carpa" y cuya cronología llega a ser mucho más reciente. En la Península Ibérica se trata principalmente de piezas atribuibles a los tipos Isorna, Miraveche y Monte Bernorio. Las dos primeras comparten los recazos exagerados del tipo Monte Sa Idda, pero se localizan principalmente en el norte (Fig. 8). Estas armas de la Edad del Hierro marcan el final de la trayectoria evolutiva de las espadas en lengua de carpa y por sus características peculiares se deben considerar, como mucho, primas lejanas dentro de la "gran familia de espadas en lengua de car- 


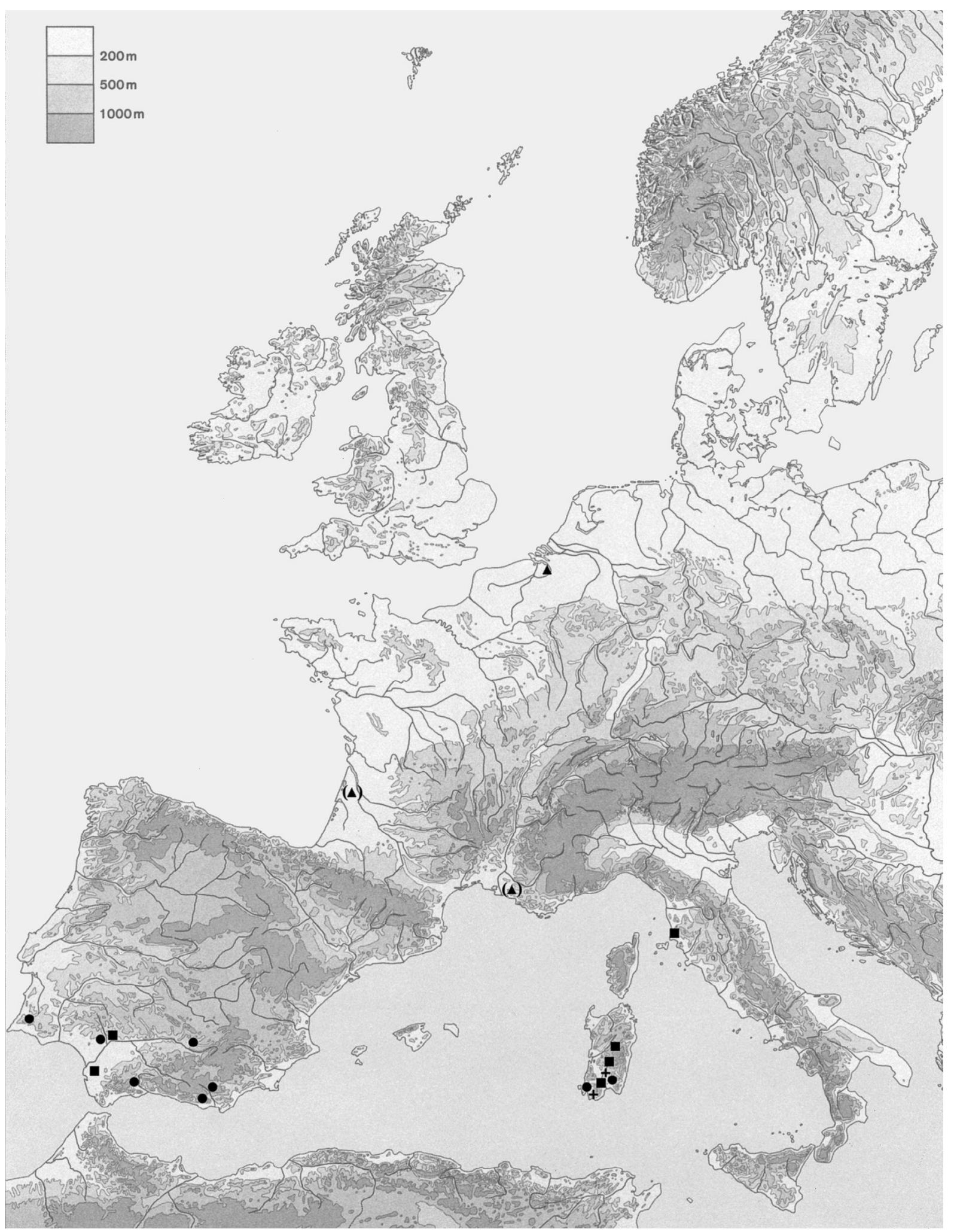

Fig. 7. Dispersión geográfica del tipo Monte Sa Idda: variante Villaverde del Río $=\mathbf{\square}$; variante Alcalá del Río $=\bullet$; variante Boom $=\boldsymbol{\Lambda}$; variante sin determinar $=\mathbf{+}-$ (según Brandherm y Burgess 2008: Fig. 7). 


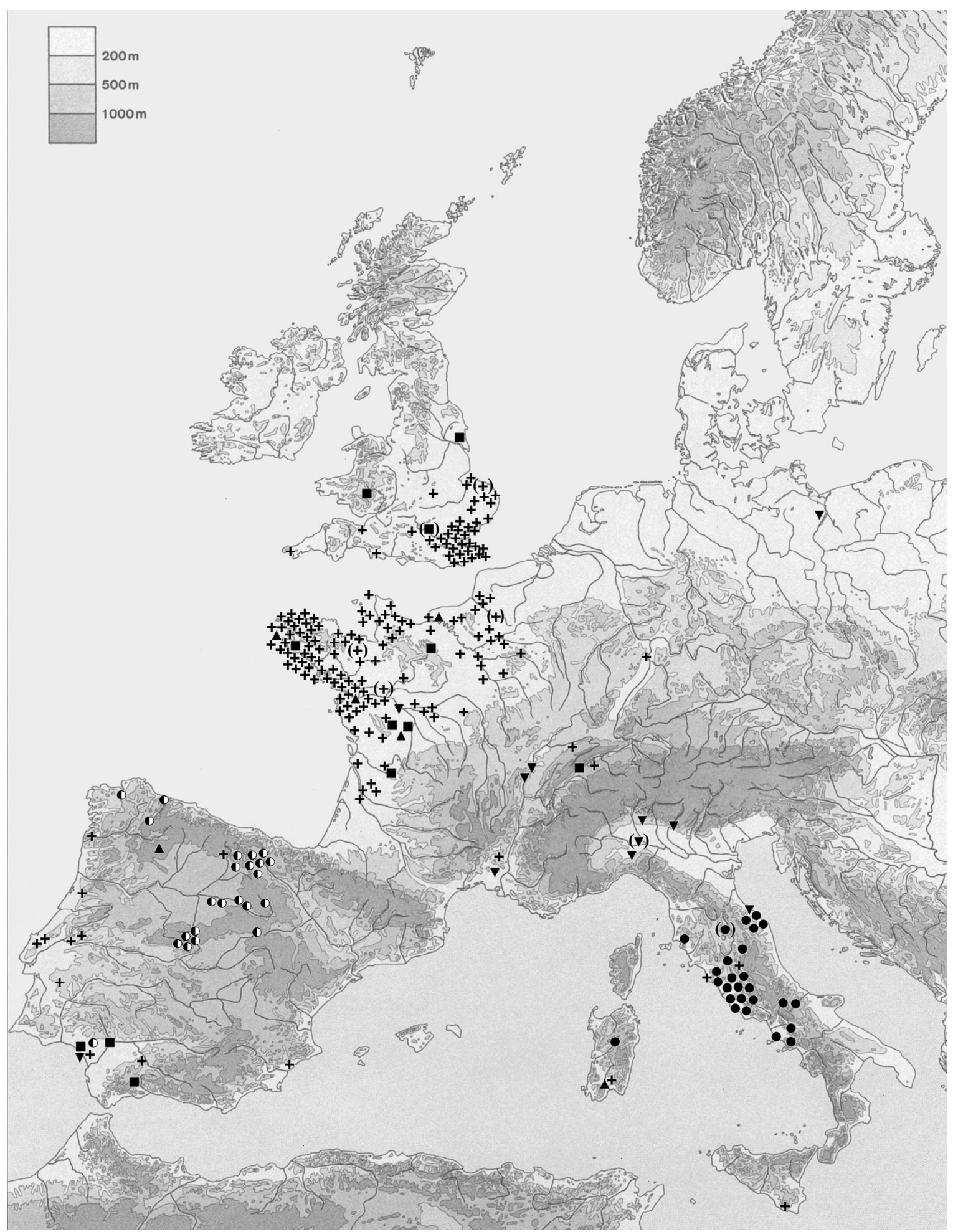

Fig. 8. Espadas en lengua de carpa con empuñaduras "transicionales" = $\mathbf{\square}$; espadas de lengüeta itálicas con hoja en lengua de carpa = ; espadas peninsulares de la Edad del Hierro con hoja en lengua de carpa $=\mathbf{O}$; espadas en lengua de carpa con empuñadura semimaciza $=\boldsymbol{\Lambda}$; espadas en lengua de carpa con empuñadura maciza $=\boldsymbol{\nabla}$; espadas en lengua de carpa de tipo indeterminado $=\boldsymbol{+}-$ (según Brandherm y Burgess 2008: Fig. 8 [actualizado]).

T. P., 67, N. ${ }^{\circ} 2$, julio-diciembre 2010, pp. 431-456, ISSN: 0082-5638 doi: $10.3989 /$ tp.2010.10049 
pa" evocada por Cowen. Por esta razón, no se suelen incluir en la definición de este grupo de armas sensu stricto.

Más estrechamente relacionado con los tres tipos canónicos de espadas en lengua de carpa -Huelva, Nantes y Monte Sa Idda- resultan las piezas de bronce con hoja en lengua de carpa y empuñadura maciza o semimaciza (Fig. 6: e). Constituyen un grupo morfológico poco homogéneo, ya que en el Bronce Final atlántico estas espadas no suelen producirse en grandes series como en Centroeuropa. Casi siempre se trata de creaciones bastante individualizadas que copian de una manera muy general los respectivos prototipos centroeuropeos, sin formar patrones morfológicos suficientemente estables para poder hablar de "tipos" propios. Consecuentemente, su dispersión en muchos casos coincide con la de los talleres de producción de espadas en lengua de carpa donde fácilmente se pudieron conocer modelos centroeuropeos de empuñadura maciza o semimaciza (Fig. 8).

\section{ASPECTOS METALÚRGICOS}

Las consideraciones acerca de la composición elemental de las aleaciones de bronce de las espadas en lengua de carpa pueden ser solamente preliminares debido al número relativamente bajo de espadas analizadas. En este grupo destacan los análisis de las piezas del tipo Huelva, en su gran mayoría efectuados por S. Rovira (1995, 2007): 49 ejemplares, provenientes principalmente de la Ría de Huelva y del depósito de Puertollano (Tab. 1). Las espadas de la Ría de Huelva muestran una composición análoga a otros objetos del mismo depósito, lo que desde el punto de vista metalúrgico testimonia su relativa homogeneidad (Rovira 1995: 34-37). Todas son bronces binarios con un $10,8 \%$ de estaño $(\mathrm{Sn})$ por término medio y una cantidad mínima de plomo $(\mathrm{Pb})-0,08 \%$ (Rovira 1995: 34, Rovira 2007: 166-171). Otra espada analizada, procedente de Bornos, también puede incluirse en este grupo, aunque carece de plomo (Rovira 1995: 38). La figura 9 (A y B)
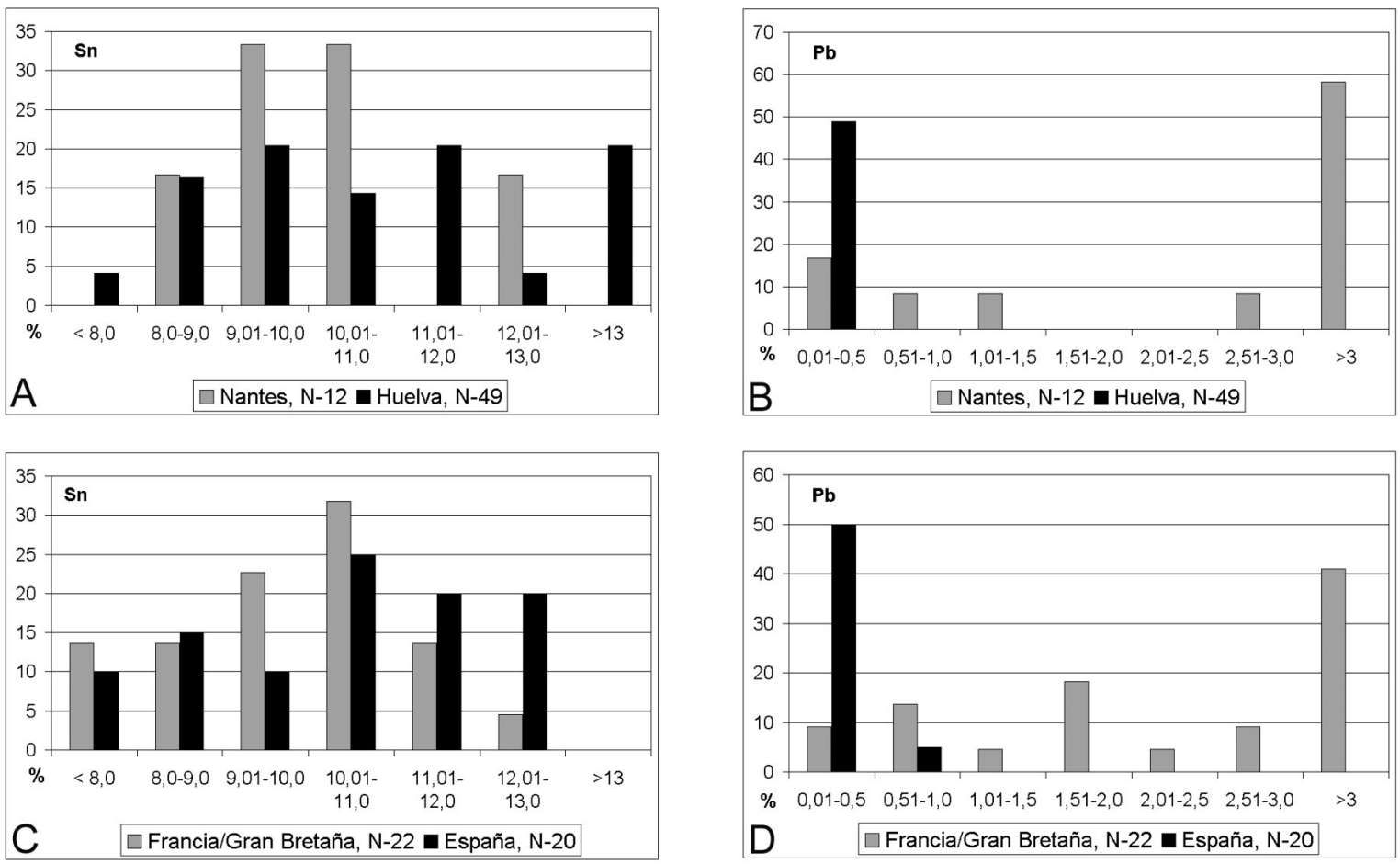

Fig. 9. Contenido de estaño y plomo en las espadas del tipo Huelva y Nantes (A y B) y de tipo indeterminado de las espadas en lengua de carpa procedentes de Francia y Gran Bretaña (C y D). 


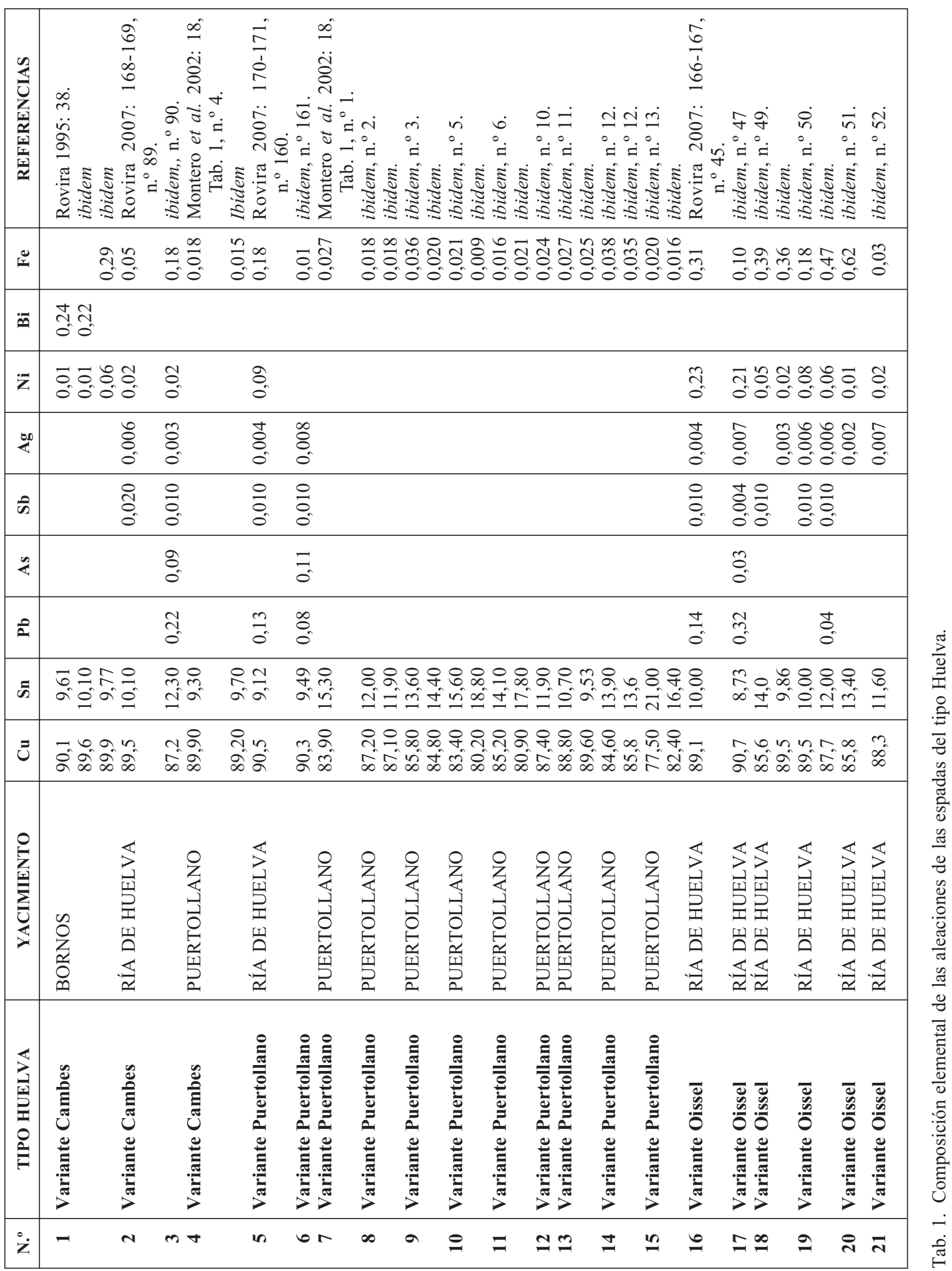

T. P., 67, N. ${ }^{\circ} 2$, julio-diciembre 2010, pp. 431-456, ISSN: 0082-5638 doi: $10.3989 /$ tp. 2010.10049 


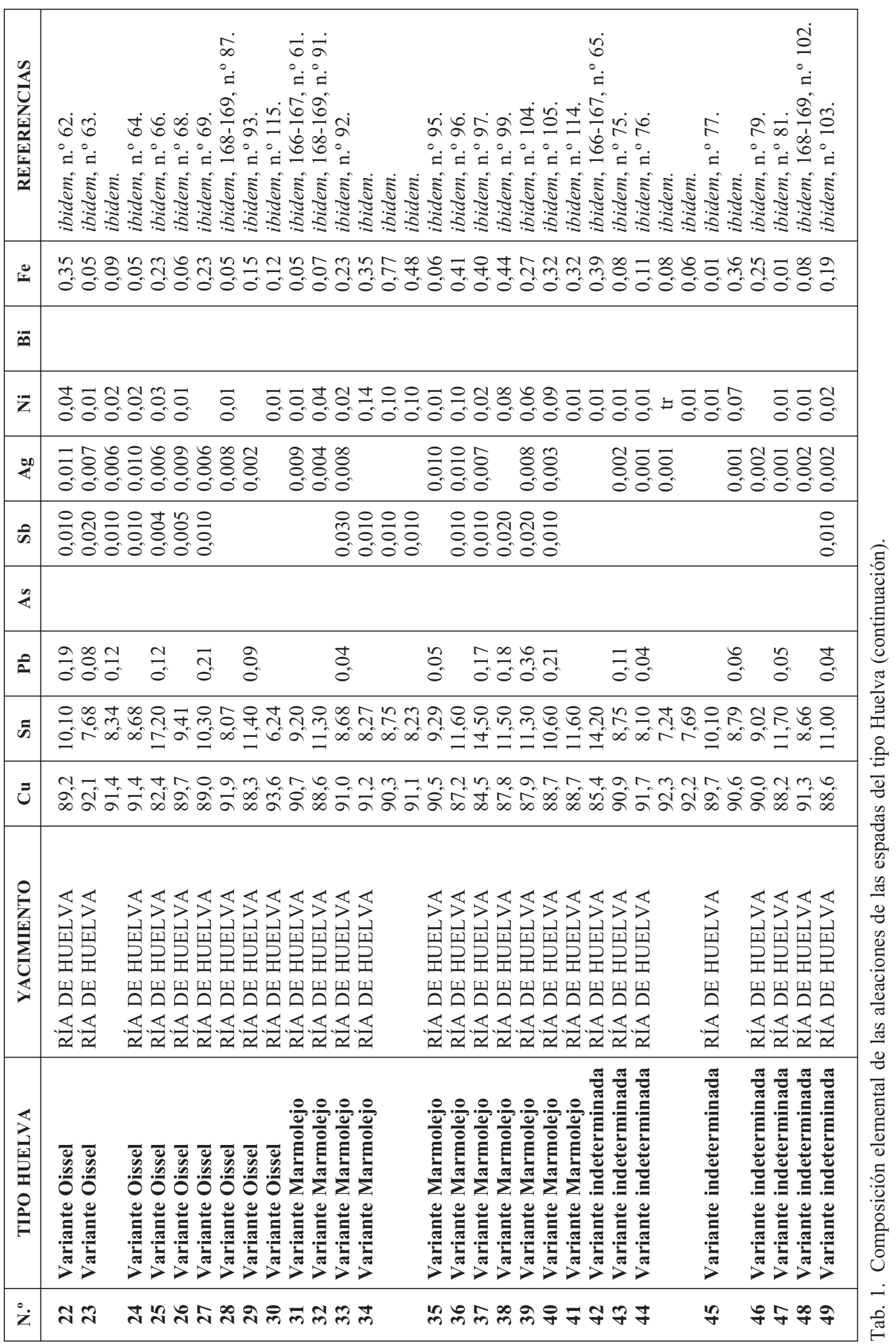

T. P., 67, N. ${ }^{\circ} 2$, julio-diciembre 2010, pp. 431-456, ISSN: 0082-5638 doi: $10.3989 /$ tp.2010.10049 
muestra la distribución del contenido de plomo y estaño de las 49 espadas del tipo Huelva. Los bronces son principalmente binarios (cobre-estaño) y, cuando se detecta plomo, no excede del $0,5 \%$ (Fig. 9: B). El contenido de estaño suele oscilar entre el 8 y $12 \%$, sin embargo, también las hay con porcentaje más alto (Fig. 9: A). Este último grupo está representado sobre todo por los ejemplares del depósito de Puertollano, algunos de los cuales superan el $17 \%$ (Tab. 1). Ello parece poco funcional, al disminuir las propiedades mecánicas de los bronces, volviéndoles más quebradizos (Rovira 2007: 156). Una explicación de este fenómeno es la probable existencia de reparaciones (Montero et al. 2002: 20).

Los análisis de las espadas del tipo Nantes procedentes de Francia muestran, por lo general, más plomo (Fig. 9: B), como el ejemplar de Brion "Grandes-Chapelles" que contiene un $5,9 \%$ y la espada de Guingamp con un 4,7\% (Tab. 2). En diferentes tomas analíticas en un ejemplar de Saint-Yrieix-sur-Charente "Vénat" el plomo oscila entre el 3,6 y el 3,8\%. Es muy probable que los demás ejemplares del conjunto también tengan este tipo de aleación, puesto que la composición elemental de bronces de todo el depósito se caracteriza por valores de plomo entre el 3,6 \% y el 8,5\% (Coffyn et al. 1981: 24). Sin embargo, no supera el $1 \%$ en algunas espadas de los depósitos de Auvers $(0,8 \%)$ y de Amiens "Le Plainseau" (0,1 \%) (Tab. 2). Las espadas del mismo tipo procedentes de Gran Bretaña también tienen mucho plomo, como los ejemplares de Eastbourne (7,8\%), Minster $(3,4 \%)$ y Leigh II $(2,3 \%)$, aunque no siempre existen las mismas pautas: en una espada de Leigh II sólo hay un $0,28 \%$ de plomo (Tab. 2). Una espada del tipo Nantes encontrada en Holanda (Nijmegen) es asimismo un bronce ternario cobre-estaño-plomo (Tab. 2). El contenido de estaño en todos los ejemplares del tipo Nantes no supera el $13 \%$ y, en la mayoría de las piezas, varía entre el 9 y el $11 \%$ (Fig. 9: A).

Una de las espadas en lengua de carpa con la empuñadura transicional procedente del río Támesis, en Inglaterra (Colquhoun y Burgess 1988: 108 Fig. 98, 672) contiene un 9,5\% de estaño y un $0,9 \%$ de plomo (Tab. 3 , n. $^{\circ} 1$ ). En comparación con las espadas del tipo Huelva, la cantidad de plomo es un poco más elevada, acercándose a las aleaciones encontradas en las espadas del tipo Nantes. Este rasgo, por lo tanto, puede confirmar que su semejanza al tipo Nantes no es sólo morfológica.

Otros tipos de espadas en lengua de carpa con datos de la composición del bronce son tres piezas del tipo Monte Sa Idda (Tab. 3, n. ${ }^{\circ}$ 2-4). Dos de la variante Villaverde del Río contienen por término medio un $13,5 \%$ de estaño y un $0,3 \%$ de plomo, mientras que el representante de la variante Alcalá del Río tiene mayor contenido de ambos (Tab. 3, n. ${ }^{\circ}$ 4). Por el contrario, dos espadas del grupo con empuñadura maciza contienen un porcentaje de plomo muy bajo (Tab. 3, n. $^{\circ} 5$ y 6). El alto contenido de estaño en la segunda pieza puede ser de la muestra tomada en el pomo (Rovira 2007: 170 n. $\left.{ }^{\circ} 199\right)$ y corresponder al proceso de sobremoldeo (Rovira 2007: 158).

No es fácil realizar comparaciones cuantitativas más exhaustivas entre espadas diferentes, analizadas con distintas técnicas y con diversas metodologías. Entre las causas que pueden influir y/o alterar los resultados destacan las técnicas analíticas cuyas limitaciones pueden introducir errores. Asimismo, es muy importante el protocolo relativo al número total de tomas analíticas y zonas muestreadas, exigibles para que los resultados sean fiables, que puede disminuir las distorsiones ocasionadas por la heterogeneidad de la propia pieza investigada (Northover y Rychner 1998: 20, 31; Montero et al. 2002: 20 y 21; Rovira 2007: 156-157). Además, algunos cambios experimentados por el objeto analizado, como la corrosión, pueden dar resultados poco representativos del estado original. No obstante, los datos analíticos, a pesar de sus limitaciones, suelen ser comparables al inscribirse en ciertas pautas (Northover y Rychner 1998: 31). Indicarían una abundancia relativa y la presencia/ausencia de los elementos, señalando simultáneamente tendencias generales entre varios tipos de las aleaciones. Por ejemplo, en el caso del plomo, primordial para diferenciar los tipos de espadas en lengua de carpa a escala europea, debido al proceso de segregación, su contenido suele ser más alto en el nervio central o en las partes más gruesas de la empuñadura que en las partes más finas y distales de la hoja (Hughes et al. 1982: 359-361; Montero et al. 2002: 20). Teniendo en consideración dichas limitaciones debemos centrarnos principalmente en su ausencia y presencia mínima o más destacada, especialmente cuando desconocemos la procedencia de la muestra y la metodología aplicada. 


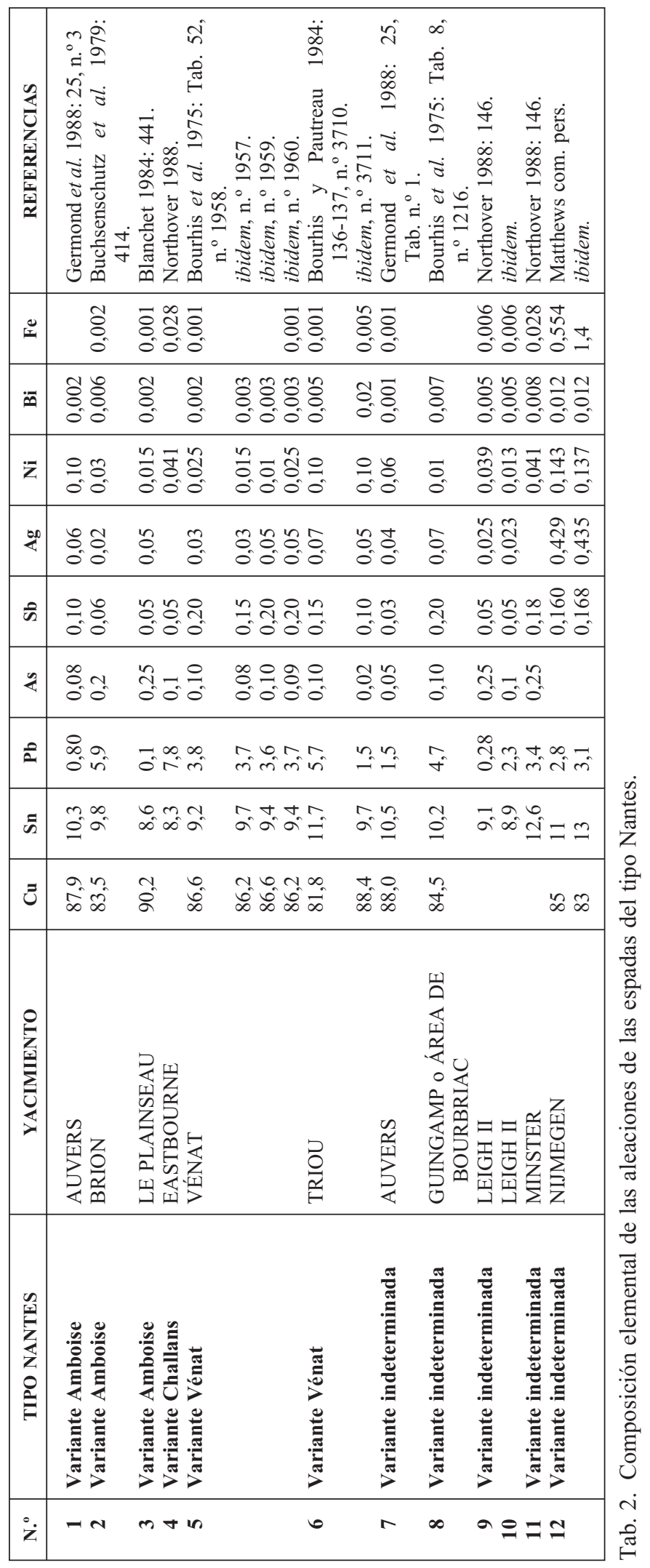

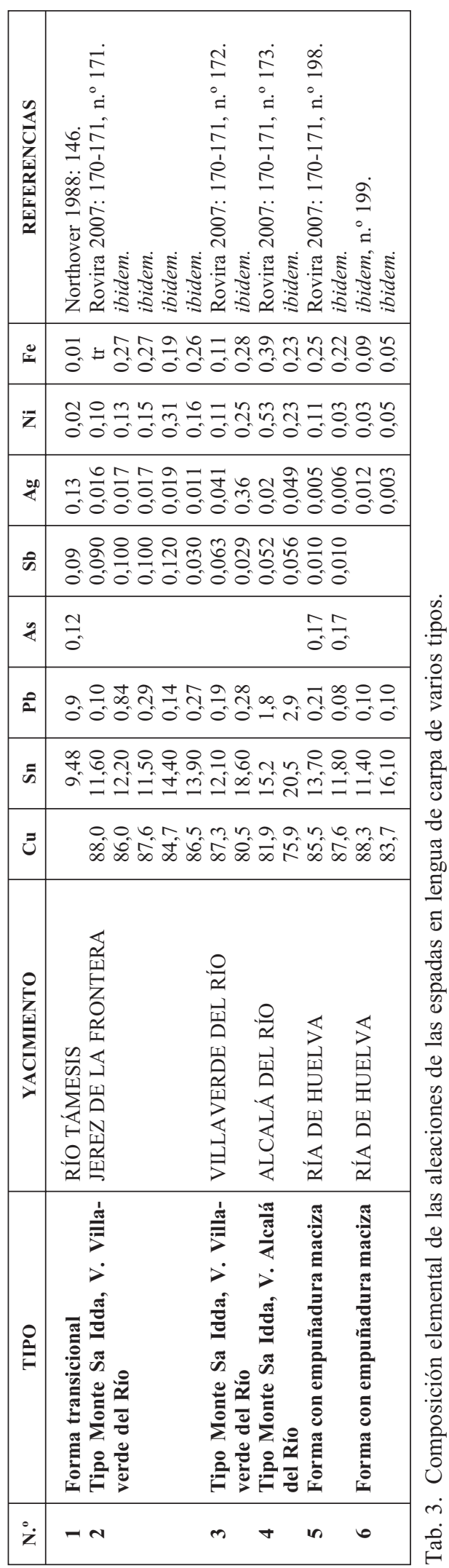

T. P., 67, N. ${ }^{\circ} 2$, julio-diciembre 2010, pp. 431-456, ISSN: 0082-5638 doi: $10.3989 /$ tp.2010.10049 
En conclusión, en todo el conjunto de espadas en lengua de carpa hemos observado que el contenido de plomo es muy diverso, lo que nos ha permitido una división en dos grupos de aleaciones: los bronces binarios y ternarios (cobre-estaño-plomo). El primer grupo corresponde claramente a las espadas del tipo Huelva, mientras que el segundo es más frecuente en las espadas del tipo Nantes. Los demás tipos tienen muy pocos datos para permitir generalizaciones, aunque los ejemplares del tipo Monte Sa Idda contienen mayor tasa de plomo que las piezas del tipo Huelva.

Los trabajos de S. Rovira (1995, 2007, Rovira y Gómez 1998) han indicado previamente las diferencias entre la metalurgia peninsular, caracterizada por los bronces binarios, y la atlántica-norpirenaica utilización frecuente de bronces con mayor contenido de plomo. Además, la comparación de S. Rovira entre la metalurgia de los bronces de la Ría de Huelva con la de otras zonas de la Península Ibérica ha demostrado diferencias en la cantidad de plomo con las regiones de la ribera del Duero (promedio de 2,6\%) y el noroeste peninsular $(19,4 \%)$, así como afinidades con las regiones del noreste peninsular y las Baleares. Ello constata que su metalurgia se relaciona más con la zona mediterránea que con la atlántica (Rovira 1995: 53, 55; Rovira 2007: 158). Las espadas del tipo Huelva también se asemejan en este aspecto con las espadas pistiliformes (Rovira 1995: 39), sugiriendo una cronología relativamente antigua.

Por otro lado, Rovira y Gómez (1999: 82) han comparado los análisis de aleaciones de varios grupos de bronces del Bronze final atlantique III / fase Ewart Park originarios de Francia e Inglaterra con la metalurgia de la Península Ibérica. En lo que se refiere a diversos tipos de espadas británicas, los autores han observado mayor contenido de plomo (tasa media del 3,8\%). Los ejemplares franceses también demuestran el uso del bronce con plomo (media del 5,3 \% Pb). En comparación con espadas y otros grupos de objetos realizados en bronce, se ha confirmado el carácter particular de los bronces peninsulares, manifestado sobre todo por la baja presencia de plomo (Rovira y Gómez 1998: 82, Rovira 2007: 158).

En general, el elevado contenido de plomo en el Bronce Final Atlántico también supone un rasgo cronológico, puesto que a lo largo de este período aumenta su cantidad en los bronces, alcanzando su auge en la transición a la Edad del Hierro. Suponiendo que este rasgo sea un indica- dor cronológico, se puede razonar que las espadas del tipo Huelva son más tempranas que las del tipo Nantes. Aunque la composición química de los bronces puede deberse entre otras razones a una diferente accesibilidad a los metales o a preferencias culturales. En el ámbito atlántico, la aparición de los bronces ternarios también se relaciona con frecuencia con la actividad del fundidor y la presencia de los depósitos de chatarra (Coffyn et al. 1981: 29; Northover 1988). De todas maneras, es muy razonable la consideración por Rovira y Gómez (1998: 88) del uso del plomo en los últimos momentos del Bronce Final en Europa atlántica como un rasgo cultural.

Finalmente, se han analizado 39 espadas en lengua de carpa de tipo indeterminable, por falta de la parte diagnóstica de la empuñadura (Tab. 4). Los primeros 20 ejemplares son espadas de la Península Ibérica (Tab. 4,1-20) y los últimos de Francia e Inglaterra. En el primer grupo prevalecen las espadas dragadas de la Ría de Huelva y una pieza de Estepa. La mayoría de estos ejemplares carece de plomo en su composición o lo tiene en porcentajes muy bajos (Fig. 9: D), como en las espadas del tipo Huelva. El contenido de estaño es diverso, siendo más frecuente el grupo con valores entre el 10 y $12 \%$ Sn (Fig. 9: C).

En cambio, las piezas halladas en Francia e Inglaterra contienen mayor porcentaje de plomo: como promedio el $4,7 \% \mathrm{~Pb}$. Además, en casi el $40 \%$ de los ejemplares supera el $3 \%$ (Fig. 9: D). El contenido de estaño es muy variado pero el grupo más abundante oscila entre el 9 y $10 \%$ (Fig. 9: C). Estos rasgos son más característicos de las espadas del tipo Nantes. Consecuentemente, podemos suponer que, por su distinta distribución, y discrepancias en la composición de las aleaciones, el primer grupo correspondería con más probabilidad a las espadas del tipo Huelva (bronces binarios), y el segundo al tipo Nantes (bronces ternarios).

\section{CONTEXTO ARQUEOLÓGICO Y LOS DEPÓSITOS DEL "COMPLEJO DE LENGUA DE CARPA"}

Las espadas en lengua de carpa proceden de contextos arqueológicos muy diversos, pero los más habituales son ambientes acuáticos, depósitos y hallazgos singulares. La mayoría de las espadas del tipo Huelva proviene de ambiente acuá- 


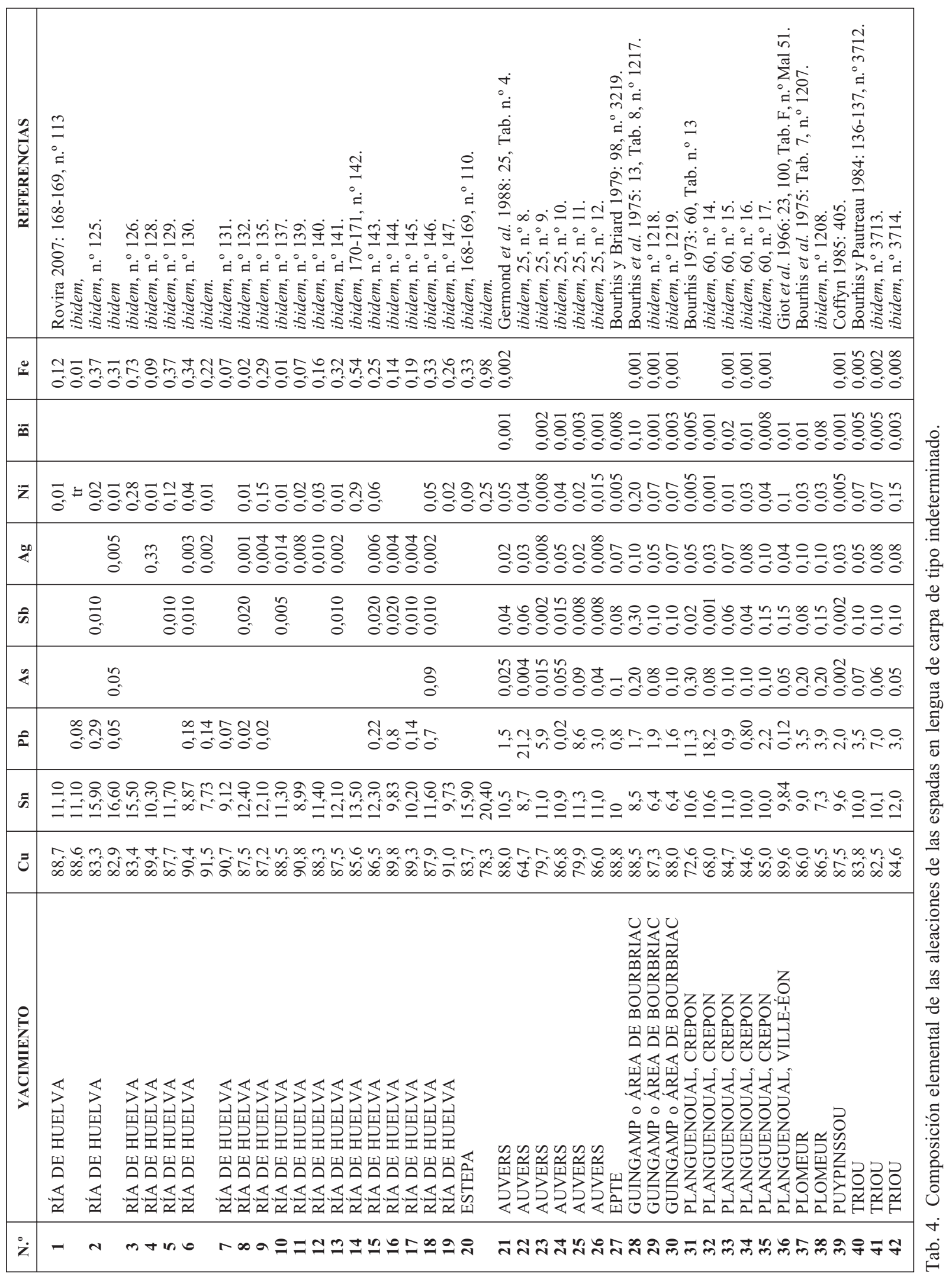

T. P., 67, N. ${ }^{\circ} 2$, julio-diciembre 2010, pp. 431-456, ISSN: 0082-5638 doi: $10.3989 /$ tp.2010.10049 
tico y solamente en dos ocasiones de depósitos en tierra, ambos hallados en la Península Ibérica: Cangas de Morrazo "Hío" (Pontevedra) y Puertollano "Camino de Santiago" (Ciudad Real). El primero, además de la espada, contiene piezas datadas en la fase Wilburton de Gran Bretaña, como las puntas de lanza de sección romboidal (Coombs 1975: 56-61; Coffyn 1985: 43, 201) y los calderos de la clase A0 (Coffyn 1985: 230; Gerloff 2010: 203-204), lo que puede sugerir su cronología relativamente temprana dentro del ámbito de espadas en lengua de carpa. El segundo depósito únicamente tiene armas, predominando las espadas del tipo Huelva, sin otras piezas que podrían indicar una cronología más precisa (e. g. puñales tipo Porto de Mos) (Coffyn 1985: 171, 207, 217-218; Fernández-García 1997: 98, 102, 113-115; Brandherm 2007: 77-86).

En cambio, la mayoría de las espadas del tipo Nantes proviene de depósitos en tierra, cuyos conjuntos presentan muchas similitudes en lo que respecta a la composición de objetos (e. g. Briard 1965: 203-225; Jockenhövel y Smolla 1975). Uno de los más destacados es precisamente este tipo de espada en lengua de carpa, por lo que este fenómeno se suele denominar el "complejo de lengua de carpa", independientemente de que los respectivos depósitos contengan o no la espada epónima. La mayoría de los objetos encontrados en los depósitos son característicos de la fase Bronze final atlantique III que es correlacionada con la fase Ewart Park de Gran Bretaña. No obstante, en muchas ocasiones están datados por nada más que la presencia de espadas en lengua de carpa, lo que impide una datación más exacta (Briard 1965: 199-225; Blanchet 1984: 308). Anteriormente, el depósito de Saint-Yrieix-sur-Charente "Vénat" constituía el límite más reciente. Se consideraba ocultado al final del siglo VIII o al principio del siglo VII a.C. (Coffyn et al. 1981: 61; Coffyn 1985: 122). Hoy en día, con los cambios en la cronología absoluta del Ha $\mathrm{C}$ también se admite su cronología anterior (Gómez de Soto 1991: 371). Entre los depósitos con espadas del tipo Nantes hay tanto aquellos que contienen objetos exclusivamente atlánticos como también conjuntos mixtos que demuestran las influencias del mundo de la cultura de Campos de Urnas (Briard 1965: 229-230; Jockenhövel y Smolla 1975: 308). Excepcionalmente, se encuentran objetos mediterráneos como los conocidos del conjunto de Saint-Yrieix-sur-Charente "Vénat".
Además, las espadas del tipo Nantes raramente aparecen fuera del ámbito atlántico. Véase, por ejemplo, el depósito mediterráneo de Decimoputzu "Monte Sa Idda" o el depósito de Campos de Urnas de Wojciechowice en Polonia (Podkowińska 1933: 116, 120).

En la tabla 5 resumimos la presencia o ausencia de los objetos más habitualmente encontrados en los depósitos del "complejo de lengua de carpa", en un total de 44 conjuntos con espadas del tipo Nantes (3). Destacan las espadas, varios tipos de puñales y cuchillos. Las espadas del tipo Ewart Park (Tab. 5, 1) son las más frecuentes. Se diferencian de las espadas en lengua de carpa por la hoja pistiliforme de sección lenticular, la morfología de la guarda y los recazos poco marcados (Briard 1965: 205; Blanchet 1981: 301; Colquhoun y Burgess 1988: 66-67). Este tipo es considerado británico, ya que ha evolucionado del tipo Wilburton, característico de la fase homónima (Burgess y Colquhoun 1988: 55, 66) (4).

En estos depósitos hay muy numerosas y diferentes puntas de lanza (Tab. 5, 2), difíciles de asimilar a algún tipo (Briard 1965: 216). Los ejemplares de base tubular decorada (Tab. 5, 3) se relacionan con la influencia británica (Blanchet 1984: 303). De otras armas, se conocen varios tipos de puñales, destacando los de cubo (Tab. 5, 4) y los de lengüeta (Tab. 5, 5) frecuentes en Gran Bretaña (Blanchet 1984: 302) (5). También son habituales en estos contextos los cuchillos de un solo filo (Tab. 5, 6), los cuchillos en lomo de puerco (6) de forma rectangular (Tab. 5, 7) y con menor frecuencia unas navajas triangulares (Briard 1965: 219).

(3) Advertimos que el análisis de los objetos asociados a las espadas del tipo Nantes es preliminar debido, principalmente, a que se basa en la bibliografía que no siempre informa con suficiente lujo de detalles. Para las referencias bibliográficas relacionadas con los depósitos: Moskal 2007; Brandherm y Burgess 2008.

(4) En la bibliografía francesa este tipo se puede encontrar bajo el nombre Ewart Park-Challans, puesto que las respectivas piezas podrían haber sido fabricadas también en la zona atlántica de Francia, lo que viene apoyado por la existencia de moldes o ejemplares mal fundidos (Coffyn et al. 1981: 52; Verney 1990: 397).

(5) Estas armas, incluidas en el grupo de los puñales por su doble filo, también pueden ser denominados cuchillos, o couteau à soie o couteau à douille en francés.

(6) Este objeto en Gran Bretaña aparece como cuchillo (hog s-back knife), mientras que en Francia suele llamarse racloire à bélière. 


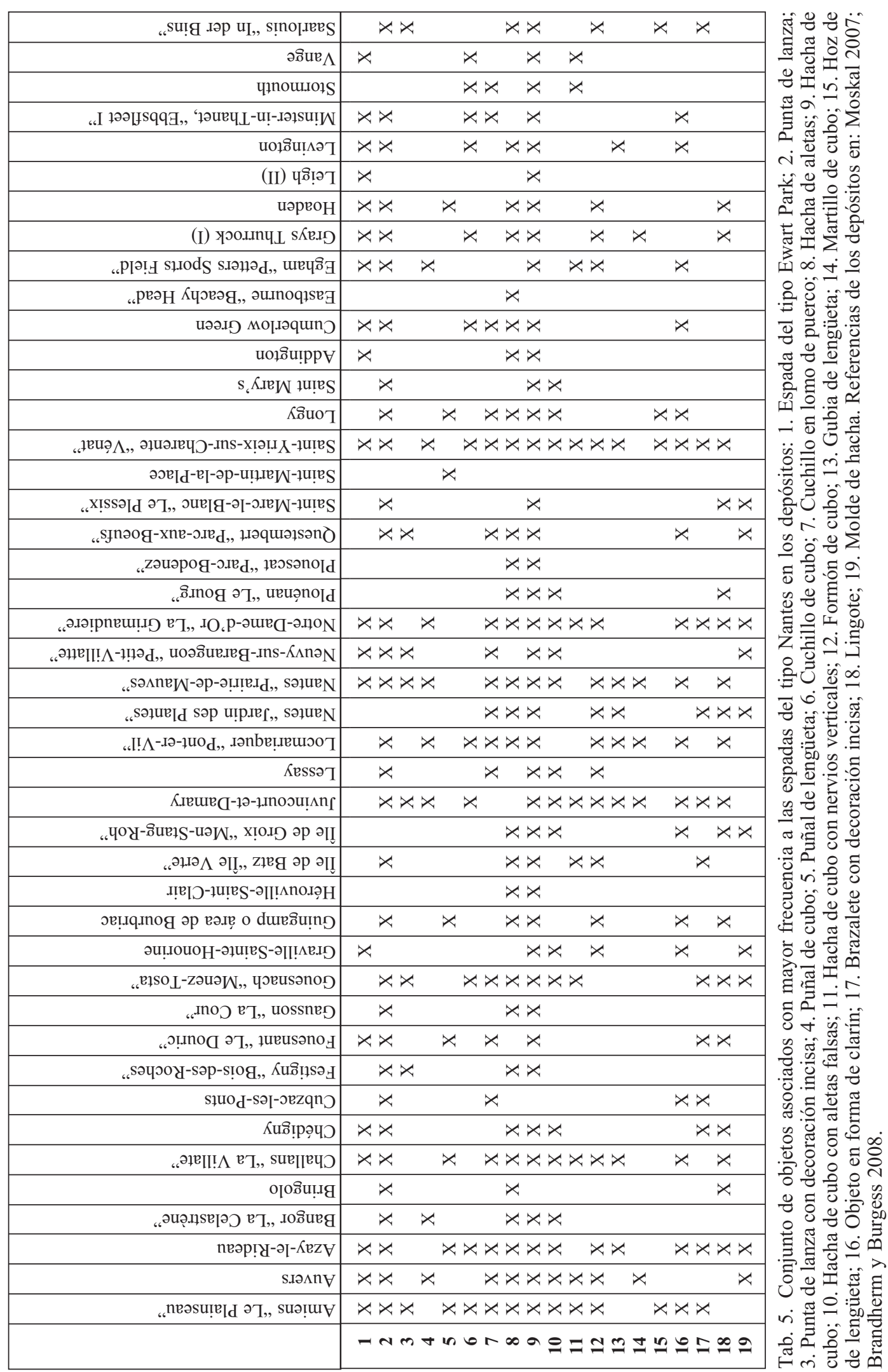

T. P., 67, N. ${ }^{\circ}$ 2, julio-diciembre 2010, pp. 431-456, ISSN: 0082-5638 doi: $10.3989 /$ tp.2010.10049 
Dentro de los útiles predominan las hachas, habitualmente de aletas (7) (Tab. 5, 8) y de cubo (Tab. 5, 9). El primer tipo procede de Europa central de la cultura de Campos de Urnas, donde es citado en los períodos anteriores. Sin embargo, se considera que en la parte atlántica de Francia, al imitar el modelo centroeuropeo, estaba producido localmente, como pueden testimoniar varios moldes (Briard 1965: 203, 211, 213; Briard y Verron 1976: 5-7; Coffyn et al. 1981: 52). Las hachas de cubo se remontan al Bronce Medio centroeuropeo, pero varios moldes demuestran que también fueron fabricadas en la zona atlántica (Briard 1965: 216; Briard y Verron 1976: 29). Constituyen el grupo atlántico las hachas Plaiseau que se caracterizan por cubos circulares con decoración de aletas falsas (Tab. 5, 10) (Briard y Verron 1976: 35-36; Blanchet 1984: 282; Coffyn 1985: 100). Frecuentemente se han encontrado hachas de cubo de tipo inglés con tres nervios (Tab. 5, 11), datadas en la fase Ewart Park (Jockenhövel y Smolla 1975: 302; Schmidt y Burgess 1981: 238). En este grupo distinguimos los tipos de Gales y Yorkshire que difieren por la sección del cubo (redonda o cuadrada) (Briard y Verron 1976: 63-65; Schmidt y Burgess 1981: 223-241). Otros útiles muy característicos de estos depósitos son los formones de cubo (Tab. 5, 12), las gubias (8) (Jockenhövel y Smolla 1975: 302-303) (Tab. 5, 13) y los martillos tubulares de sección cuadrada (Tab. 5, 14). Estos últimos en Centroeuropa y Francia ya eran conocidos desde el Bronce Medio (Jockenhövel y Smolla 1975: 302; Blanchet 1984: 303). También aparecen hoces de lengüeta (Tab. 5,15$)$ y de cubo con una anilla.

De tipo atlántico son los objetos en forma de clarín (9) (Tab. 5, 16) encontrados con mayor reiteración en los depósitos de Francia y de Inglaterra, posiblemente pertenecientes a bocados de caballo (Briard 1965: 219; Blanchet 1984: 306; Coffyn 1985: 100). En los adornos los brazaletes son más comunes. Sobre todo se distinguen las formas con decoración incisa (Tab. 5, 17) cuyos prototipos se originan en la cultura de Campos de Urnas (Briard 1965: 225; Jockenhövel y Smolla 1975: 306). Finalmente, dentro de estos depósitos aparecen con frecuencia lingotes (Tab. 5, 18),

(7) Fr. hache à ailerons; con mayor frecuencia: hache à ailerons subterminaux.

(8) Fr. tranchette.

(9) Ing. bugle-shaped object. moldes, principalmente de hachas (Tab. 5, 19), y otros objetos relacionados con la actividad metalúrgica, como numerosa chatarra. A propósito, hemos de resaltar que muchos de los objetos descritos, conservados parcialmente, en realidad también formaban parte de ella. Para poder sacar conclusiones más pormenorizadas sobre la variabilidad en la composición de los depósitos del "complejo en lengua de carpa", tanto en términos cronológicos como espaciales, sería necesario cuantificar los distintos tipos de objetos en los respectivos conjuntos, información de la cual en muchos casos no disponemos (véase Boulud y Fily 2009: 286-288).

Vale la pena prestar atención igualmente a las espadas en lengua de carpa fragmentadas que carecen de lengüeta y por eso son inclasificables (Moskal 2007; Brandherm y Burgess 2008: lista $21)$. Es muy probable que estos fragmentos hubieran pertenecido a la familia de las espadas en lengua de carpa dadas sus características morfológicas y distribución. Otro factor que puede apoyar esta atribución es su contexto. En este sentido, nos referimos principalmente a los fragmentos procedentes de depósitos, que forman la gran mayoría de dichos hallazgos y, además, muchos aparecieron en los típicos depósitos del "complejo de lengua de carpa". Cuentan con el mismo conjunto de piezas que se asocian con las espadas del tipo Nantes (Tab. 5). Efectivamente, las espadas del tipo Nantes aparecieron en 26 de los 103 depósitos analizados. En conclusión, esta observación y cierta homogeneidad de los depósitos nos permite razonar que, por lo menos, los fragmentos que provienen de los depósitos del "complejo de lengua de carpa" con mucha probabilidad corresponderían a espadas del tipo Nantes (10).

(10) En el caso de los depósitos británicos es muy apropiada la hipótesis de que dichos fragmentos pertenecen a espadas en lengua de carpa, puesto que en la fase Ewart Park en las Islas Británicas las espadas dominantes (Ewart Park) presentaban otra sección (lenticular) (Colquhoun y Burgess 1988: 55-111). Las únicas espadas que poseen una sección parecida a las espadas en lengua de carpa se encuentran entre las de antenas que, aunque se fechan en la misma fase, resultan excepcionales (Colquhoun y Burgess 1988: 122-123). Mayor problema causan los ejemplares procedentes de Francia, donde había más tipos de espadas con la sección similar a la de las espadas en lengua de carpa. Primero, en la transición del Bronze final atlantique II al Bronze final atlantique III no se pueden clasificar con seguridad los fragmentos de hojas, ya que podrían ser también de espadas pistiliformes con fuerte nervio central (Briard 1965: 190-192, 198). Segundo, existen espadas de empuñadura maciza con la sección parecida, como p. e. el tipo Mörigen o algunas piezas 


\section{CONCLUSIONES}

En primer lugar hay que destacar que, hoy por hoy, difícilmente se puede mantener una tipogénesis de las espadas en lengua de carpa a partir de las espadas pistiliformes tardías del tipo Saint Nazaire, como en su día fue defendido por Cowen (1956), ya que aquellas resultan contemporáneas con las primeras espadas tipo Huelva o incluso posiblemente posteriores a ellas. El origen exacto de estas últimas es difícil de establecer, pero parece evidente que intervienen tanto elementos heredados de las primeras espadas pistiliformes en Occidente como la morfología de los estoques tardíos de los inicios del Bronce Final (Brandherm 2007: 74). La evolución interna de las espadas en lengua de carpa partiendo del tipo Huelva hacia los tipos Nantes y Monte Sa Idda tampoco queda muy clara en todos su pasos, aunque se puede identificar un grupo de piezas con empuñaduras "transicionales". Su posible papel en este proceso resulta tan difícil de verificar como su posición cronológica con relación a dichos tipos.

En cuanto a la metalurgia, los dos tipos principales -Huelva y Nantes- difieren en el contenido de plomo, inscribiéndose a la vez en el patrón metalúrgico propio de su ámbito geográfico. El tipo Huelva claramente representa el uso de un bronce binario utilizado con mayor frecuencia en el marco mediterráneo, mientras que el tipo Nantes refleja la metalurgia con bronce ternario, característica de las regiones de la Francia atlántica e Inglaterra.

Por lo que respecta al contexto de los hallazgos, las espadas del tipo Huelva provienen principalmente de ambientes acuáticos y raramente forman parte de contextos cerrados. En cambio, los ejemplares del tipo Nantes frecuentemente aparecen en depósitos, con cierta homogeneidad en su distribución y contenido. En último caso, el propio contexto puede servir como otro punto de partida para clasificar en el tipo Nantes los fragmentos indeterminados de las espadas en lengua de carpa.

En cualquier caso hemos de resaltar que la inclusión de la Península Ibérica en el área de dis-

con empuñadura de antenas. Ambos aparecen además en depósitos con espadas en lengua de carpa como p. e. Deville-lésRouen, Crossac, Saint-Yrieix-sur-Charente "Vénat" y Wojciechowice. No obstante, constituyen un porcentaje muy bajo entre los hallazgos de la Francia atlántica. persión del "complejo de lengua de carpa" por parte de algunos autores se debe mucho menos a la realidad del registro arqueológico que a la ficción de una continuada uniformidad cultural del Bronce Final atlántico durante los primeros siglos del primer milenio a. C. (Brandherm 2007: 78; Burgess y O'Connor 2008: 55-58). En realidad, en estos momentos el sur peninsular ya había caído bajo la creciente influencia fenicia, formando cada vez menos parte del complejo cultural del Bronce Atlántico, dando lugar a unos procesos de transformación que también iban a afectar la mitad norte de la Península y últimamente incluso el mundo norpirenaico.

\section{AGRADECIMIENTOS}

Muchos colegas nos han ayudado con información sobre hallazgos inéditos o publicados en lugares poco accesibles y queremos agradecerles su inestimable ayuda. También debemos nuestra gratitud a los compañeros que han sacrificado su tiempo para discutir con nosotros detalles sobre la tipología y metalurgia de estas piezas. En particular agradecemos a Wojciech Blajer, Colin Burgess, Marek Gedl, Consuelo Mata, Steve Matthews, Renaud Nallier y Brendan O'Connor. Finalmente agradecemos a los dos evaluadores anónimos sus comentarios que nos han ayudado mucho a mejorar la versión final de nuestra contribución.

\section{BIBLIOGRAFÍA}

Almagro Basch, M. 1940: "El hallazgo de la Ría de Huelva y el final de la Edad del Bronce en el occidente de Europa". Ampurias 2: 85-143.

Bastien, G. 1966: "Quelques objets inédits de l'âge du Bronze prevenant des dragages de la Loire à la Ville-aux-Dames et à Amboise (I.-et-L.)". Bulletin de la Société Préhistorique Française 63: CCLXCCLXVI.

Begemann, F.; Schmitt-Strecker, S.; Pernicka, E. y Lo Schiavo, F. 2001: "Chemical composition and lead isotopy of copper and bronze from Nuragic Sardinia". European Journal of Archaeology 4: 43-85.

Billard, C.; Desard, B. y Lajoie, G. 1993: "Eléments sur le peuplement de la basse vallée de la Seine à partir de l'étude de matériaux de dragage". Revue archéologique de l'Ouest 10: 55-87. 
Blanchet, J.-C. 1984: Les prèmiers metallurgistes en Picardie et dans le Nord de la France. Mémoires de la Société Préhistorique Française 17. Société Préhistorique Française. Paris.

Boulud, S. y Fily, M. 2009: "Les dépôts métalliques de l'extrême fin du Bronze final en Bretagne: nouvelle évaluation des données à la lumière des découvertes récente". En De l'âge du Bronze à l'âge du Fer en France et en Europe occidentale ( $X^{e}-V I I^{e}$ siècle av. J.-C.) La moyenne vallée du Rhône aux âges $d u$ Fer. Actes $d u$ XXXe colloque international de l'A.F.E.A.F., co-organisé avec l'A.P.R.A.B. (SaintRoman-en-Gal, 26-28 mai 2006). Revue Archéologique de l'Est, supplément 27. Dijon: 283-298.

Bourhis, J.R. 1984: “Analyses des cuivres et bronzes de Picardie et du Nord de la France". En Paleometalurgie de la France Atlantique. Age du Bronze (I). Travaux du Laboratoire d'Anthropologie Préhistorique. Rennes: 23-43.

Bourhis, J.R. y Briard, J. 1979: Analyses spetrographiques d'objects préhistorique et antiques, Quatrieme serie. Travaux du Laboratoire d'Anthropologie Préhistorique. Rennes.

Bourhis, J.; Giot, P.-R. y Briard, J. 1975: Analyses spetrographiques d'objects préhistorique et antiques. III serie. Travaux du Laboratoire d'Anthropologie Préhistorique. Rennes.

Bourhis, J. y Pautreau, J.-P. 1984: “Analyses des bronzes du depôt de Triou”. En Paleometalurgie de la France Atlantique. Age du Bronze (I). Travaux du Laboratoire d'Anthropologie Préhistorique. Rennes: $135-137$.

Brandherm, D. 2007a: Las espadas del Bronce Final en la Península Ibérica y Baleares. Prähistorische Bronzefunde IV, 16. Franz Steiner. Stuttgart.

Brandherm, D. 2007b: "Swords by numbers". En C. Burgess, P. Topping y F. Lynch (eds.): Beyond Stonehenge. Essays on the Bronze Age in honour of Colin Burgess. Oxbow Books. Oxford: 288-300.

Brandherm, D. 2008: "Erneut zur Datierung der ältesten griechischen und phönizischen Importkeramik auf der Iberischen Halbinsel". Madrider Mitteilungen 49: 115-144.

Brandherm, D. y Burgess, C. 2008: “Carp's-tongue problems". En F. Ferse, B. Knoche, J. Graefe, M. Hohlbein, K. Schierhold, C. Siemann, M. Uckelmann y G. Woltermann (eds.): Durch die Zeiten... Festschrift für Albrecht Jockenhövel zum 65. Geburtstag. Internationale Archäologie, Studia honoraria 28. Marie Leidorf. Rahden: 133-168.

Brandherm, D. y Sicherl, B. 2001: "Überlegungen zur Schwertproduktion der späten Urnenfelderzeit Bemerkungen zur Herstellung späturnenfelderzeitlicher Vollgriffschwerter anhand zweier Beispiele von nördlich und südlich der Alpen". Archäologisches Korrespondenzblatt 31: 223-241.
Briard, J. 1965: Les dépôts bretons et l'âge du Bronze atlantique. Becdelière. Rennes.

Briard, J. 1966: Dépôts de l'âge du Bronze de Bretagne. La Prairie de Mauves à Nantes. Travaux du Laboratoire d'Anthropologie Prehistorique. Rennes.

Briard, J. 1971: "Le dépôt du Jardin des Plantes à Nantes (Bronze Final)". Études Préhistoriques et Protohistoriques des Pays de la Loire 2: 3-35.

Briard, J.; Le Roux, C.-T. y Onnée, Y. 1973: “Les dépôts de Planguenoual et le Bronze final de Côtes-du-Nord". Annales de Bretagne 70: 35-59.

Briard, J. y Verron, G. 1976: Typologie des objets de l'âge du Bronze en France, fasc. IV: haches (2). Société Préhistorique Française. Paris.

Brown, M.A. y Blin-Stoyle, A.E. 1959: "A sample analysis of British Middle and Late Bronze Age material, using optical spectrometry". Proceedings of the Prehistoric Society 25: 189-208.

Buchsenschutz, O.; Willaume, M. y Gablin, P. 1979: "Le site Bronze final - Premier âge du Fer des Grandes-Chapelles à Brion (Indre)". Bulletin de la Société Préhistoriques Française 76: 408-420.

Burgess, C. y O'Connor, B. 2008: "Iberia, the Atlantic Bronze Age and the Mediterranean". En S. Celestino, N. Rafel y X.-L. Armada (eds.): Contacto cultural entre el Mediterráneo y el Atlántico (siglos XII-VIII ane). La precolonización a debate. Serie Arqueológica 11. CSIC. Madrid: 41-58.

Coffyn, A. 1985: Le Bronze Final atlantique dans la Péninsule Ibérique. Publications du Centre Pierre Paris 11. Boccard. Paris.

Coffyn, A.; Gómez, J. y Mohen, J.-P. 1981: L'apogée du bronze atlantique. Le dépôt de Vénat. L'âge du bronze en France 1. Picard. Paris.

Colquhoun, I. y Burgess, C.B. 1988: The Swords of Britain. Prähistorische Bronzefunde IV, 5. C.H. Beck. München.

Coombs, D. 1975: "Bronze Age weapon hoards in Britain". Archeologia Atlantica 1: 49-81.

Cordier, G. y Gruet, M. 1975: "L'âge du Bronze et le premier âge du Fer en Anjou". Gallia Préhistoire 18: 157-287.

Cowen, J.D. 1956: "Les origines des épées de bronze du type à langue de carpe". En Actas de la IV Sesión de los Congresos Internacionales de Ciencias Prehistóricas y Protohistóricas, Madrid 1954: 639-642. Zaragoza.

Cowen, J.D. 1971: “A striking maritime distribution pattern". Proceedings of the Prehistoric Society 37: 154-166.

Fernández García, S. 1997: “Los puñales tipo 'Porto de Mos' en el Bronce Final de la Península Ibérica". Complutum 8: 97-124.

Gallay, G. 1988: Die mittel- und spätbronze-sowie ältereisenzeitlichen Bronzedolche in Frankreich und auf den britischen Kanalinseln. Prähistorische Bronzefunde VI, 7. C.H. Beck. München. 
Gerloff, S. 2010: Atlantic Cauldrons and Buckets of the Late Bronze and Early Iron Ages in Western Europe. Prähistorische Bronzefunde II, 18. Franz Steiner. Stuttgart.

Germond, G.; Gómez, J.; Verron, G. y Bourhis, J.-R. 1988: "Nouvelles recherches sur le dépôt d'Auvers, Manche (Bronze Final III)". Bulletin de la Société Préhistorique Française 85: 15-31.

Giardino, C. 1995: Il Mediterraneo Occidentale fra XIV ed VIII secolo a. C. Cercie minerarie e metallurgice. British Archaeological Reports International Series 612. Tempus Reparatum. Oxford.

Giot, P.-R.; Bourhis, J. y Briard, J. 1966: Analyses spectrographiques d'objets préhistorique et antiques, I série. Travaux du Laboratoire d'Anthropologie Préhistorique. Rennes.

Gómez de Soto, J. 1991: "Le fondeur, le trafiquant et les cuisiniers. La broche d'Amathonte de Chypre et la chronologie absolue du Bronze Final Atlantique". En Ch. Chevillot y A. Coffyn (eds.): L'Âge du Bronze Atlantique. Ses faciès de l'Ecosse à l'Andalousie et leurs relations avec le Bronze Continental et la Méditerranée. Actes du $1^{\text {er }}$ colloque du Parc Archéologique de Beynac. Association des Musées du Sarladais. Beynac: 369-373.

Hughes, M.J.; Northover, J.P. y Staniaszek, B.E.P. 1982: "Problems in the analysis of leaded bronze alloys in ancient artefacts". Oxford Journal of Archaeology 1: 359-364.

Jiménez Ávila, J. 2002: La toréutica orientalizante en la Península Ibérica. Bibliotheca Archaeologica Hispana 16, Studia Hispano-Phoenicia 2. Real Academia de la Historia. Madrid.

Jockenhövel, A. 1980: Die Rasiermesser in Westeuropa. Prähistorische Bronzefunde VIII, 3. C.H. Beck. München.

Jockenhövel, A. y Smolla, G. 1975: "Le dépôt de Juvincourt-Damary (Aisne)”. Gallia Préhistoire 18: 289-309.

Mederos Martín, A. 2008: "Las espadas de tipo Huelva y los inicios de la presencia fenicia en Occidente durante el Bronce Final IIC-IIIA, 1150-950 a.C.". Cuadernos de Prehistoria y Arqueología de la Universidad Autónoma de Madrid 34: 41-75.

Meijide Cameselle, G. 1988: Las espadas del Bronce Final en la Península Ibérica. Arqueohistórica 1. Universidad de Santiago de Compostela. Santiago de Compostela.

Montero Ruiz, I.; Fernández Rodríguez, M.; Gómez Tubio, B. y Ontalba Salamanca, M.A. 2002: "Espadas y puñales del Bronce Final: el depósito de armas de Puertollano (Ciudad Real)". Gladius 22: 5-28.

Moskal, M. 2007: "Późnobrązowe miecze z językiem karpia na terenie Europy". En J. Chochorowski (ed.): Studia nad epoka brazu i wczesna epokq żelaza. Księga poświęcona Profesoriwi Markowi
Gedlowi na pięćdziesięciolecie pracy w Uniwersytecie Jagiellońskim. Wydawnictwo Uniwersytetu Jagiellońskiego. Kraków: 465-531.

Needham, S.; Ramsey, C.B.; Coombs, D.; Cartwright, C. y Pettitt, P. 1997: “An independent chronology for British Bronze Age metalwork: The results of the Oxford Radiocarbon Accelerator Programme". Archaeological Journal 154: 55-107.

Northover, P. 1988: "The analysis and metallurgy of British Bronze Age Swords". En I. Colquhoun y C.B. Burgess: The Swords of Britain. Prähistorische Bronzefunde IV, 5. C.H. Beck. München: 130-146.

Northover, J.P. y Rychner, V. 1998: "Bronze analysis: experience of a comparative programme". En C. Mordant, M. Pernot y V. Rychner (eds.): L'atelier du bronzier en Europe du XXe au VIII siècle avant notre ère. Actes du colloque international "Bronze 96” de Neuchâtel et Dijon 1. Éditions du Comité des Travaux Historiques et Scientifiques et du Centre de recherches sur les techniques gréco-romaines de l'Université de Bourgogne. Paris: 19-40.

Podkowińska, Z. 1933: "Miecze brązowe z Wojciechowic w pow. Jędrzejowskim w woj. Kieleckim". Światowit 15: 116-168.

Quilliec, B. 2007a: L'épée atlantique: échanges et prestige au Bronze final. Mémoires de la Société Préhistorique Française 42. Société Préhistorique Française. Paris.

Quilliec, B. 2007b: Vida y muerte de una espada atlántica del Bronce Final en Europa. Reconstrucción de los procesos de fabricación, uso y destrucción. Complutum 18: 93-117.

Rovira Llorens, S. 1995: "Estudio arqueometalúrgico del depósito de la Ría de Huelva”. En M. Ruiz-Gálvez Priego (ed.): Ritos de paso y puntos de paso. La Ría de Huelva en el mundo del Bronce Final europeo. Complutum Extra 5. Servicio de Publicaciones de la Universidad Complutense. Madrid: 33-57.

Rovira Llorens, S. 2007: "Las espadas del Bronce final de la Península Ibérica: estudio arqueometalúrgico". En D. Brandherm: Las espadas del Bronce Final en la Península Ibérica y Baleares. Prähistorische Bronzefunde IV, 16. Franz Steiner. Stuttgart: 155-175.

Rovira, S. y Gómez-Ramos, P. 1998: "The Ría de Huelva hoard and the Late Bronze Age metalwork: a statistical approach". En C. Mordant, M. Pernot y V. Rychner (eds.): L'atelier du bronzier en Europe du XX $X^{e}$ au VIII ${ }^{e}$ siècle avant notre ère. Actes du colloque international "Bronze 96" de Neuchâtel et Dijon 1. Éditions du Comité des Travaux Historiques et Scientifiques et du Centre de recherches sur les techniques gréco-romaines de l'Université de Bourgogne. Paris: 81-90.

Ruiz-Gálvez Priego, M. 1984: La Península Ibérica y sus relaciones con el círculo cultural atlántico. Editorial de la Universidad Complutense. Madrid. 
Ruiz-Gálvez Priego, M. 1986: "Navegación y comercio entre el Atlántico y el Mediterráneo a fines de la Edad del Bronce". Trabajos de Prehistoria 43: 9-42.

Ruiz-Gálvez Priego, M. (ed.) 1995: Ritos de paso y puntos de paso. La Ría de Huelva en el mundo del Bronce Final europeo. Complutum Extra 5. Servicio de Publicaciones de la Universidad Complutense. Madrid.

Savory, H.N. 1949: "The Atlantic Bronze Age in south-west Europe". Proceedings of the Prehistoric Society 15: 128-155.
Schmidt, P.K. y Burgess, C.B. 1981: The Axes of Scotland and Northern England. Prähistorische Bronzefunde IX, 7. C.H. Beck. München.

Schüle, W. 1969: Die Meseta-Kulturen der Iberischen Halbinsel. Madrider Forschungen 3. Walter de Gruyter. Berlin.

Taramelli, A. 1921: "Il ripostiglio dei bronzi nuragici di Monte Sa Idda di Decimoputzu (Cagliari)". Monumenti antichi dei Lincei 27: 6-107.

Verney, A. 1990: "Le dépôt de Challans (Vendée)". Bulletin de la Société Préhistorique Française 87: 396-417. 